Antje Töpel (Mannheim)

\title{
Methoden zur Erstellung von Bedeutungsparaphrasenangaben
}

\author{
$1 \quad$ Einleitung \\ 2 Terminologie \\ 3 Theoretische Einflussfaktoren \\ 3.1 Linguistische Theorien \\ 3.2 Lexikografische Methoden \\ 3.3 Typen von Bedeutungs- \\ paraphrasenangaben
}

\author{
$4 \quad$ Das Beispiel elexiko - \\ Vorgehen beim Erstellen \\ der semantischen \\ Paraphrase \\ 5 Ausblick \\ 6 Literaturangaben
}

\begin{abstract}
Once a new word or a new meaning is added to a monolingual dictionary, the lexicographer is to provide a definition of this item. This paper focuses on the methodological challenges in writing such definitions. After a short discussion of the central terminology (method and definition), the article describes factors which inform this process: linguistic theories, linguistic and lexicographical methods, and types of definitions. Using the example of elexiko, a dictionary project of the Institute for the German language (IDS) in Mannheim, Germany, the paper finally showcases the compilation of definitions in a monolingual online dictionary of contemporary German.
\end{abstract}

Keywords: einsprachiges Wörterbuch, lexikographische Methoden, Definition, Bedeutungserläuterung, lexikografischer Prozess

\section{Einleitung}

Wenn ein Wort in ein Bedeutungswörterbuch aufgenommen wird, muss unter anderem die Semantik beschrieben werden, es muss also eine semantische Paraphrase oder Bedeutungsparaphrasenangabe erstellt werden. Diesem Thema widmet sich der vorliegende Beitrag, wobei die methodischen Aspekte beim Verfassen von Paraphrasen im Mittelpunkt stehen. ${ }^{1}$ Dafür werden zunächst die Begriffe der Bedeutungsparaphrasenangabe und der Methode geklärt (Abschnitt 2). Für das Erstellen

1 Im Kontext der Bedeutungserläuterung bestehen daneben noch weitere Teilbereiche, für die methodische Aspekte diskutiert werden. Moerdijk (2003, 285ff.), Svensén (2009, 205ff.) und Reichmann (2012, 341ff.) beschreiben Methoden zur Ordnung von Lesarten, Wiegand (2010, 254ff.) geht auf Methoden zur Ermittlung von Bedeutungsparaphrasen ein. Cramer (2011) führt Methoden zur automatischen Extraktion semantischer Paraphrasen aus. 
semantischer Paraphrasen existieren bis dato allerdings keine allgemein anerkannten Methoden. Auch die metalexikografische Forschungsliteratur zum Thema ist überschaubar. Aus diesem Grund werden in Abschnitt 3 die drei Themenbereiche, die das Erstellen einer Paraphrase in theoretischer Hinsicht besonders stark beeinflussen - linguistische Theorien, linguistische und lexikografische Methoden und die Typen semantischer Paraphrasen - näher vorgestellt. Die drei Bereiche können dabei nicht komplett unabhängig voneinander beschrieben werden, da sie sich gegenseitig beeinflussen. Beispielsweise wirken sich die linguistischen Theorien auf die Typen von Bedeutungserläuterungen aus. Daneben bestehen beim Erstellen semantischer Paraphrasen noch weitere Einflussfaktoren, wie etwa der Wörterbuchtyp. Solche Faktoren werden an dieser Stelle nur im Kontext der unterschiedlichen Paraphrasentypen angerissen, da sich diese Darstellung auf monolinguale, synchrone, allgemeinsprachliche Wörterbücher konzentriert. ${ }^{2}$ Abschnitt 4 zeigt schließlich am Beispiel von elexiko, wie in der Praxis eines monolingualen Onlinewörterbuchs des Gegenwartsdeutschen beim Verfassen der Bedeutungserläuterung vorgegangen wird. Ein Ausblick (Abschnitt 5) vervollständigt die Darstellung.

\section{Terminologie}

Der von Wiegand geprägte Begriff der Bedeutungsparaphrasenangabe wird im WLWF wie folgt beschrieben: „Bedeutungsparaphrasenangaben sind die zentralen, aber nicht isoliert zu betrachtenden Angaben in allen Wörterbüchern, für die das Prinzip der semantischen Kodierung gilt [...]. Sie kommen vor allem in Wörterbuchartikeln vor, und der paraphrasierte, vorher erwähnte Ausdruck ist meistens das Lemmazeichen [...].“ (WLWF, 648). Hier werden die Bezeichnungen „(semantische) Paraphrase“, „Bedeutungsparaphrase“ und „Bedeutungserläuterung“ synonym zum Begriff „Bedeutungsparaphrasenangabe“ verwendet. ${ }^{3}$

Weiterhin ist der Methodenbegriff näher zu betrachten. Auch dabei kann auf die Arbeiten Wiegands zurückgegriffen werden: „Eine Methode ist eine geordnete Menge von Handlungsanweisungen, deren Befolgung in mindestens einer geordneten Reihenfolge und unter Beachtung aller methodenzugehöriger Korrektheitsbedingungen erfolgen muss, damit das gewünschte Ergebnis erhältlich ist.“ (Wiegand 2010, 251). Diese kurze Charakterisierung ist für metalexikografische Zwecke vollkommen aus-

2 Auf Modelle der Bedeutungsbeschreibung in der zweisprachigen Lexikografie geht Knipf-Komlósi (2000) am Beispiel eines deutsch-ungarischen Wörterbuchs ein.

3 Um die Benennung der Angabe, die Wiegand als Bedeutungsparaphrasenangabe bezeichnet, gibt es einen seit Jahrzehnten andauernden Streit in Lexikografie und Metalexikografie, der sich im Kern um die Verwendung des Begriffes „Definition“ für diese Angabe dreht. Stellvertretend für die Diskussion sei an dieser Stelle auf Wiegand (1989) verwiesen. 
reichend. In Bezug auf das Verfassen von semantischen Paraphrasen geht es also um die Darstellung der Arbeitsschritte, die zu einer Bedeutungsparaphrasenangabe führen.

\section{Theoretische Einflussfaktoren}

\subsection{Linguistische Theorien}

Wie eine semantische Paraphrase erstellt wird, ist auf einer ersten theoretischen Ebene von linguistischen Theorien abhängig. Zwar hat bisher keine sprachwissenschaftliche Theorie eine konkrete Methode zum Verfassen von Bedeutungsparaphrasenangaben für Wörterbücher vorgelegt, jedoch spiegeln sich in den konkreten semantischen Paraphrasen bestimmte theoretische Ansätze wider, wie die Merkmalssemantik oder der Prototypenansatz. ${ }^{4}$

Die Vertreter der Merkmalssemantik übertrugen in den 1960-er Jahren die Arbeiten aus der Phonologie, die Lautstruktur eines Wortes in Phoneme zu zerteilen, auf die Semantik. ${ }^{5}$ Analog sollte die Bedeutungsseite eines Lexems in einzelne Merkmale (Seme) aufgespalten werden. Ziel dieser Komponentenanalyse war es, ein im Umfang begrenztes Set an universell gültigen, nicht weiter teilbaren Semen zu erstellen, mit deren Hilfe der gesamte Wortschatz beschrieben werden kann. Als Ausgangspunkt wurden einzelne Wortfelder (wie Verwandtschaftsbezeichnungen oder Bezeichnungen für Sitzgelegenheiten) analysiert. Die Komponentenanalyse sollte die Mitglieder der Wortfelder (wie Vater, Mutter, Sohn, Tochter, Kind) in ihren Unterschieden, aber auch in ihren Beziehungen untereinander erfassen. Heringer (1968) zeigt dies am Beispiel einiger Sitzmöbel (cf. Tab. 1).

\footnotetext{
4 Herbst/Klotz gehen in ihrer Einschätzung sogar noch weiter: „Vorrangige Funktion der Definitionen in einem Wörterbucheintrag ist die Darstellung von Wortbedeutungen. Dabei ist anzumerken, dass die semantische Forschung im Rahmen der theoretischen Linguistik kein Modell zur Beschreibung von Bedeutungen entwickelt hat, das für lexikografische Zwecke unmittelbar nutzbar wäre, sondern dass im Gegenteil die eigentliche deskriptive Leistung im Hinblick auf die semantische Analyse des Wortschatzes einer Sprache eher - wenn auch vielleicht zum Teil auf unbefriedigender theoretischer Basis - durch Wörterbücher erfolgt.“ (Herbst/Klotz 2003, 33).

5 Als einführende Lektüre zur Merkmalssemantik bieten sich beispielsweise Lutzeier (1985, 91ff.), Helbig (2002, 86f.) oder Elsen (2014, 95ff.) an.
} 
Tab. 1: Merkmalsanalyse für das Wortfeld „Sitzgelegenheit“ (cf. Heringer 1968, 227)

\begin{tabular}{lccccc}
\hline & $\begin{array}{c}\text { mit } \\
\text { Rückenlehne }\end{array}$ & $\begin{array}{c}\text { mit } \\
\text { Armlehne }\end{array}$ & $\begin{array}{c}\text { für eine } \\
\text { Person }\end{array}$ & $\begin{array}{c}\text { Möbel zum } \\
\text { Sitzen }\end{array}$ & $\begin{array}{c}\text { mit } \\
\text { Polsterung }\end{array}$ \\
\hline Stuhl & + & - & + & + & + \\
Sessel & + & + & + & + & + \\
Hocker & - & - & + & + & \\
Bank & & & - & + & + \\
Sofa & + & + & - & + & + \\
\hline
\end{tabular}

Parallelen zur Komponentenanalyse finden sich in der logischen Definition (cf. Abschnitt 3.3). Auch hier wird das Lexem durch distinktive Merkmale in seiner Bedeutung von ähnlichen Lexemen abgegrenzt, durch die Wahl eines Hyperonyms aber auch relativ zu anderen Lexemen beschrieben und innerhalb seines Wortfeldes eingeordnet. Am Beispiel einiger Lexeme des Wortfeldes „Sitzgelegenheiten“ zeigt sich eindrücklich, wie die einzelnen Seme der Komponentenanalyse ('mit Rückenlehne', 'mit Armlehne', 'für eine Person', 'Möbel zum Sitzen' und 'mit Polsterung') in den Bedeutungsparaphrasenangaben von Duden online durchscheinen: Stuhl 'mit vier Beinen, einer Rückenlehne und gelegentlich Armlehnen versehenes Sitzmöbel für eine Person' (Duden online), ${ }^{7}$ Sessel 'mit Rückenlehne, gewöhnlich auch mit Armlehnen versehenes, meist weich gepolstertes, bequemes Sitzmöbel (für eine Person); Polstersessel' (Duden online), Hocker '[stuhlhohes] Sitzmöbel ohne Lehne für eine Person' (Duden online), Bank 'Sitzgelegenheit aus Holz, Stein o.̈̈., die mehreren Personen nebeneinander Platz bietet' (Duden online) und Sofa 'gepolstertes Sitzmöbel mit Rückenlehne und Armlehnen, dessen Sitzfläche für mehrere Personen Platz bietet' (Duden online). ${ }^{8}$

6 Vorhandene Seme erhalten ein „+“, nicht vorhandene Seme ein „,-“, irrelevante Seme werden weggelassen. Heringer (1968) wandelt das ursprünglich von Pottier (1978) verwendete Beispiel (in der deutschen Version erst 1978, 68) leicht ab. Außerdem ist seine Schreibweise eine andere als die hier benutzte: Stuhl $\mathrm{S}_{1}=\mathrm{s}_{1} \overline{\mathrm{s}}_{2} \mathrm{~s}_{3} \mathrm{~s}_{4}$, Sessel $\mathrm{S}_{2}=\mathrm{s}_{1} \mathrm{~s}_{2} \mathrm{~s}_{3} \mathrm{~s}_{4} \mathrm{~s}_{5}$, Hocker $\mathrm{S}_{3}=\overline{\mathrm{s}}_{1} \overline{\mathrm{s}}_{2} \mathrm{~s}_{3} \mathrm{~s}_{4}$, Bank $\mathrm{S}_{4}=\overline{\mathrm{s}}_{3} \mathrm{~s}_{4}$, Sofa $\mathrm{S}_{5}=\mathrm{s}_{1} \mathrm{~s}_{2}$ $\overline{\mathrm{s}}_{3} \mathrm{~s}_{4} \mathrm{~s}_{5}$, wobei $\mathrm{s}_{1}=$ 'mit Rückenlehne', $\mathrm{s}_{2}=$ 'mit Armlehne', $\mathrm{s}_{3}=$ 'für eine Person', $\mathrm{s}_{4}=$ 'Möbel zum Sitzen' und $s_{5}=$ 'mit Polsterung'. Auch hier werden irrelevante Seme weggelassen, negative erhalten einen hochgestellten Querstrich (cf. Heringer 1968, 227). Für eine noch umfangreichere Komponentenanalyse unterschiedlicher Sitzgelegenheiten und Trinkgefäße cf. Ayto (1983, 91ff.).

7 Bei polysemen Wörtern wird nur die Lesart zitiert, die hier von Bedeutung ist.

8 An dieser Stelle muss erwähnt werden, dass eine klassische Merkmalsanalyse keine Bedeutungserläuterung zum Ergebnis hat, sondern in vielen Fällen eine stark formalisierte Ausdrucksweise, die für Benutzer von Wörterbüchern vollkommen unbrauchbar wäre. Der Lexikograf kann eine Merkmals- 
Eine weitere linguistische Theorie, die im Kontext der Erstellung von Bedeutungsparaphrasenangaben eine Rolle spielen kann, ist der Prototypenansatz. ${ }^{9} \mathrm{Er}$ geht davon aus, dass es für jede Kategorie (wie z.B. „Vogel“) bessere Vertreter (die Prototypen, bei den Vögeln etwa das Rotkehlchen für den US-amerikanischen Raum) und schlechtere Vertreter (im Beispiel „Vogel“ etwa der Pinguin oder der Strauß) gibt (cf. Abb. 1):

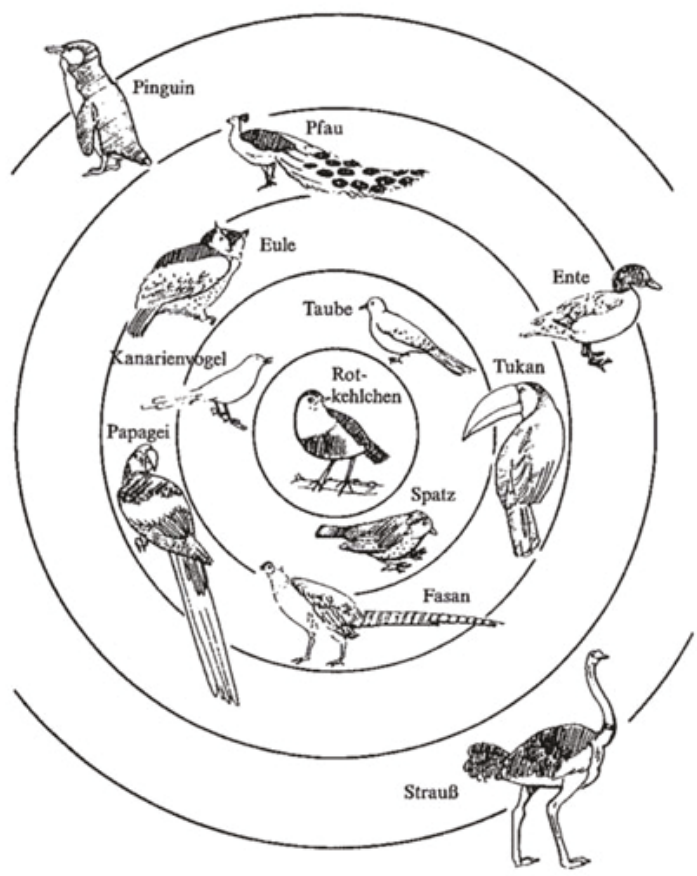

Abb. 1: Gute und schlechte Vertreter für die Kategorie „Vogel“ (Aitchison 1997, 68)

Der Prototypenansatz spiegelt sich ebenfalls in einem bestimmten Typ von Bedeutungsparaphrase wider - der extensionalen Erläuterung (cf. Abschnitt 3.3). Die dort aufgezählten Hyponyme sind, wenn nicht alle Kohyponyme aufgelistet werden können, in dem jeweiligen Kulturkreis typische Vertreter ihrer Klasse, die über möglichst viele Eigenschaften des Prototypen verfügen. Sie können auch durch Heckenausdrücke (wie typischerweise oder im Allgemeinen) eingeleitet werden. So nennt

analyse also im besten Fall als Ausgangspunkt für seine Formulierung einsetzen. Zum Verhältnis von semantischer Paraphrase und Komponentenanalyse cf. auch Schmidt (1981, 78ff.).

9 Für eine Einführung in den Prototypenansatz cf. Aitchison (1997, 65ff.), Helbig (2002, 276ff.) oder Elsen (2014, 101ff.), zur Anwendung im Kontext der Bedeutungsparaphrase cf. Atkins/Rundell (2008, 417ff.). 
die Bedeutungsparaphrase von Hausarbeit bei Duden online die typischen Hausarbeiten (Putzen, Waschen, Kochen): Hausarbeit 'im Haushalt anfallende Arbeit (wie Putzen, Waschen, Kochen)' (Duden online). Hausarbeiten, die im Bedeutungsspektrum weniger zentral angesiedelt sind, wie das Wechseln von Glühbirnen oder das Polieren von Möbeln, finden keine Erwähnung. Gleiches gilt für die semantische Paraphrase beim Beispiel Pflanze in elexiko: 'Mit Pflanze bezeichnet man ein Lebewesen (z.B. einen Baum, eine Blume, Gras), das mithilfe seiner grünen Farbe und des Sonnenlichts in einem Stoffwechselprozess (Fotosynthese) Energie produzieren kann.' (elexiko). Die Hyponyme, die hier erwähnt werden, Baum, Blume und Gras, sind typische Pflanzen, die im Zentrum des Bedeutungsspektrums stehen. Periphere Vertreter (wie Moos oder Kaktus) bleiben unerwähnt. Das Anführen prototypischer Hyponyme oder Beispiele in der Bedeutungsparaphrasenangabe kann dazu beitragen, dass der Wörterbuchbenutzer schneller die Verbindung zwischen dem nachgeschlagenen Lexem und dessen außersprachlichem Referenten herstellt, was das Ziel einer semantischen Paraphrase ist. ${ }^{10}$

In der Wörterbuchpraxis sind beide theoretische Ansätze - die Komponentenanalyse und die Prototypensemantik - miteinander verbunden, denn häufig finden sich beide in einer semantischen Paraphrase vereint, wie bei Bett, Lesart 'Möbelstück': 'Mit Bett bezeichnet man ein meist rechteckiges, flaches Möbelstück mit einer (weichen) Auflage, das man benutzt, wenn man ausruhen oder schlafen will.' (elexiko). Allerdings bestehen auch kulturspezifische Vorlieben für eine der beiden linguistischen Theorien: Während die semantischen Paraphrasen in französischen Wörterbüchern eher in der Tradition der Merkmalssemantik stehen, sind englische Wörterbücher stärker vom Prototypenansatz beeinflusst (cf. Rothe 2001, 85ff. und Rothe 2004, 75f.).

\subsection{Lexikografische Methoden}

Für das Erstellen von Bedeutungsparaphrasenangaben existiert bisher keine ausgearbeitete Methodologie - im Gegensatz etwa zur Wörterbuchbenutzungsforschung, für die bereits eine umfangreiche Darstellung der Methodik vorliegt (cf. Wiegand 1998, 568-1025). Die Literatur zum Thema „Bedeutungsparaphrase“ klammert das Thema überwiegend aus. Das liegt zum einen natürlich daran, dass die Wörterbuchbenutzungsforschung im Vergleich zum Verfassen einzelner lexikografischer Angaben ein viel breiteres Gebiet ist, das sich zum anderen auch viel leichter auf ein bereits vorhandenes Methodenrepertoire einer anderen Wissenschaft, nämlich der empiri-

10 Die Bedeutungsparaphrase „enumerates only the most important semantic features of the defined lexical unit, which suffice to differentiate it from other units“ (Zgusta 1971, 253). Im Gegensatz dazu sollen enzyklopädische Definitionen möglichst umfassend ausfallen. 
schen Sozialforschung, stützen kann. Jedoch nutzt auch die praktische Lexikografie Erkenntnisse aus anderen Forschungsbereichen: „Bei der lexikographischen Tätigkeit wird, und zwar determiniert durch den jeweiligen Typ des zu erarbeitenden Nachschlagewerkes, auf Ergebnisse, Methoden und Theorien aus verschiedenen Wissenschaften zurückgegriffen.“(Wiegand 1984, 559).

Die Methoden, die in der Literatur im Zusammenhang mit der Erstellung lexikografischer Angaben genannt werden, beziehen sich - mit Ausnahme der Darstellung bei Reichmann (2012), der sich jedoch auf historische Wörterbücher bezieht - nicht speziell auf die Bedeutungsparaphrasenangabe, sondern betreffen im konkretesten Fall mehrere lexikografische Angaben. Es werden also keine spezifischen Methoden für das Verfassen semantischer Paraphrasen beschrieben, sondern allgemeinere lexikografische oder linguistische Methoden. Diese Methoden werden im Folgenden vom Allgemeinen zum Speziellen vorgestellt. ${ }^{11}$

Auf einer universellen Ebene kann der Prozess der Wörterbucherstellung - und darin eingeschlossen das Verfassen einer Bedeutungsparaphrasenangabe - als kombinierte Methode aus Analyse und Synthese verstanden werden, wie es Atkins/ Rundell (2008) auch grafisch präsentieren (cf. Abb. 2):

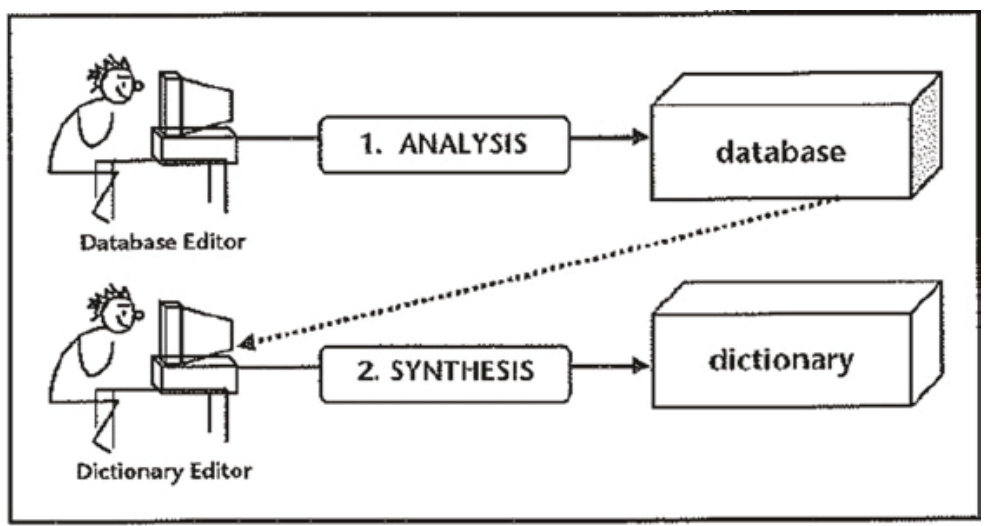

Abb. 2: Vom Korpus zum einsprachigen Wörterbuch (Atkins/Rundell 2008, 98)

Bezogen auf die semantischen Paraphrasen dient der Schritt der Analyse dazu, die Einzelwortbedeutungen oder Lesartenbedeutungen $\mathrm{zu}$ finden (cf. Atkins/Rundell 2008, 100ff.). In der Synthese entsteht dann die Bedeutungsparaphrasenangabe, im

11 Im Zusammenhang mit der Gewinnung lexikografischer Angaben wird auch der Einsatz automatisierter Verfahren diskutiert. Im Gegensatz zu den Beispielangaben oder grammatischen Angaben ist die maschinelle Erstellung von Bedeutungsparaphrasenangaben jedoch noch nicht praxistauglich (cf. Rundell/Kilgarriff 2011, 279). 
Idealfall inhaltlich und formal abgestimmt auf ihre Funktion (Rezeption/Produktion) und ihre Zielgruppe (cf. Atkins/Rundell 2008, 405ff.). ${ }^{12}$

Etwas differenzierter ist die von Schaeder (1981) entwickelte primär-induktive Methodik, die zwar allgemein linguistischer Natur ist, sich aber auch auf das Erstellen semantischer Paraphrasen anwenden lässt. Die primär-induktive Methodik kann in mehrere Teilschritte untergliedert werden (cf. Schaeder 1981, 45ff.). Im ersten Schritt werden die implizit vorhandenen Vorannahmen über den Gegenstand Sprache und das Forschungsobjekt expliziert. Der zweite Teilschritt, die Beschreibung, setzt sich aus mehreren Handlungen zusammen: der Beobachtung, dem innersprachlichen Vergleichen und Identifizieren, der Delimination oder Segmentation und der Klassifikation. Er ist mit der Analyse bei Atkins/Rundell (2008) vergleichbar. Im dritten Schritt, der Argumentation, geht es darum, eine Behauptung durch Daten zu belegen und damit eine Generalisierung zu schaffen, die empirisch gestützt ist. An letzter Stelle stehen schließlich Beschreibung und Erklärung, die in etwa der Synthese bei Atkins/Rundell (2008) entspricht.

Bergenholtz/Mugdan (1990) beziehen sich in ihrem Beitrag ausschließlich auf den Akt der Datenerhebung, dafür beschreiben sie die unterschiedlichen Methoden speziell unter dem Aspekt der lexikografischen Angaben. Dabei haben sie „vorwiegend semantische und grammatische Angaben sowie die Markierung von Stilebene, regionaler Verbreitung oder Häufigkeit im Auge“ (Bergenholtz/Mugdan 1990, 1612), weisen jedoch darauf hin, dass sich nicht ,jede Methode in gleicher Weise für alle Arten von lexikographischen Angaben“ (Bergenholtz/Mugdan 1990, 1611f.) eignet. Als Methoden der Datenerhebung betrachten sie die Auswertung vorliegender Beschreibungen, die Introspektion, die Informantenbefragung, die Belegsammlung und das Korpus. Als bereits existierende Beschreibungen, die für die Datenerhebung infrage kommen, nennen Bergenholtz/Mugdan (1990) andere Wörterbücher, Grammatiken sowie Untersuchungen von Einzelproblemen. Im Kontext der Bedeutungsparaphrasenangabe sind vorhandene Wörterbücher für Lexikografen unabhängig von der Art des zu beschreibenden Lemmas von Bedeutung. In bestimmten Wortschatzbereichen wie Funktionswörtern, Wortbildungselementen, Mehrwortverbindungen oder Neologismen können sich jedoch auch Grammatiken oder Einzeldarstellungen anbieten. Allerdings müssen dem Lexikografen die Einzeldarstellungen auch bekannt sein und er muss sie sinnvoll in den Wörterbuchartikel integrieren können. Auch der Zeitaspekt ist an dieser Stelle nicht zu vernachlässigen. Der Hauptkritikpunkt von Bergenholtz/Mugdan (1990) ist der Umgang der Wörterbuchredaktionen mit ihren Quellen - meist bleibt unklar, welche Werke auf welche Weise genutzt wurden (cf. Bergenholtz/Mugdan 1990, 1612ff.). Weiterhin bemängeln sie das ungeprüfte Übernehmen von Daten aus Sekundärquellen, das zu Fehlern führen kann.

12 Zur Anpassung von Bedeutungserläuterungen an Wörterbuchnutzer und Wörterbuchfunktionen cf. Nielsen (2011). 
Als zweite Methode der Datenerhebung steht den Lexikografen die Introspektion bzw. die eigene Sprachkompetenz zur Verfügung, die sie dafür nutzen können, „beobachtete Sprachdaten zu bewerten, vorliegende Sprachbeschreibungen zu begutachten und selbst Sprachdaten bereitzustellen“ (Bergenholtz/Mugdan 1990, 1613). Für die Erstellung semantischer Paraphrasen ist die Sprachkompetenz des Lexikografen natürlich unabdingbar. Auch in Bezug auf die Introspektion plädieren Bergenholtz/Mugdan (1990) für eine Offenlegung, wie mit ungrammatischen Sprachdaten umgegangen wird und auf welche Art Markierungen wie „selten“ vergeben werden. Als Hauptproblem introspektiv gewonnener Daten nennen sie den möglichen Widerspruch zum tatsächlichen Sprachgebrauch, weshalb sie schlussfolgern: „umfassende Wörterbücher für relativ gut erforschte Sprachen erfordern andere Methoden“ (Bergenholtz/Mugdan 1990, 1615).

Eine weitere Methode ist die Informantenbefragung, die auch als Multiintrospektion bezeichnet wird. Hier können mehrere Sprecher z.B. vorgegebene Sprachdaten nach bestimmten Kriterien beurteilen, Merkmale bei der semantischen Beschreibung von Wörtern bewerten oder vorgegebene Äußerungen selbst ergänzen oder umformen. Im Kontext der Wörterbucharbeit wird die Informantenbefragung selten eingesetzt und wenn, ist die oft geringe Zahl an Befragten problematisch. Für das Verfassen von Bedeutungserläuterungen ist diese Methode ebenfalls nicht praktikabel, lässt man „Minibefragungen“ unter Kollegen außer Acht. Weiterhin bestehen vielfältige Mängel in der Durchführung solcher Befragungen (cf. Bergenholtz/Mugdan 1990, 1616ff.).

Als vierte Methode nennen Bergenholtz/Mugdan (1990) die Belegsammlung, die auf systematisch ausgewerteten Quellen und zufälligen Funden basieren kann. Sie dient in der Wörterbucharbeit vor allem dazu, reale Sprachdaten in Form von Belegen in den Wörterbuchartikel integrieren zu können. Deshalb spielt sie beim Erstellen von Bedeutungsparaphrasen eher eine untergeordnete Rolle, etwa wenn bestimmte sprachliche Ausdrücke in die Paraphrase übernommen werden (cf. Reichmann 2012, 224ff.). Außerdem muss bedacht werden, dass Belegsammlungen stärker das Auffällige und nicht das Übliche verzeichnen. Zudem ermöglichen sie keinerlei statistisch gesicherte Aussagen über die Häufigkeit sprachlicher Erscheinungen (cf. Bergenholtz/Mugdan 1990, 1619).

Die fünfte Methode der Datenerhebung bildet die Auswertung eines Korpus. Die Aussagen von Bergenholtz/Mugdan (1990) zu diesem Thema können allerdings nur noch unter einem historischen Blickwinkel gelesen werden, denn diese Methode spielt in der heutigen Lexikografie eine übergeordnete Rolle. ${ }^{13}$

13 So sprechen Bergenholtz/Mugdan beispielsweise davon, dass „Textkorpora in der lexikographischen Arbeit noch nicht sehr häufig benutzt“ (Bergenholtz/Mugdan 1990,1620) werden und dass die „Korpusmethode noch nicht allgemein anerkannt“ (Bergenholtz/Mugdan 1990, 1620) ist. Weiterhin entkräften sie Vorbehalte, die zu dieser Zeit gegen die Korpusauswertung bestehen, beispielsweise zu ungrammatischen Äußerungen in Korpora oder zu Verallgemeinerungen von Aussagen, die aufgrund 
In der allgemeinen Theorie der Lexikografie, die Wiegand in den 1980er Jahren entwickelt, gehören die anzuwendenden lexikografischen Methoden zur Teiltheorie C (Theorie der lexikographischen Spracherforschung), die sich aus der Theorie der Datenerhebung und der Theorie der Datenaufbereitung sowie einer Theorie zum Einsatz des Computers in beiden Bereichen zusammensetzt (cf. Wiegand 1983, 46) (cf. Abb. 3). In der Teiltheorie D (Theorie der lexikographischen Sprachbeschreibung), genauer in der Texttheorie für lexikographische Texte, wird untersucht, welche Strukturen lexikografische Texte haben sollten (cf. Wiegand 1983, 46ff.).

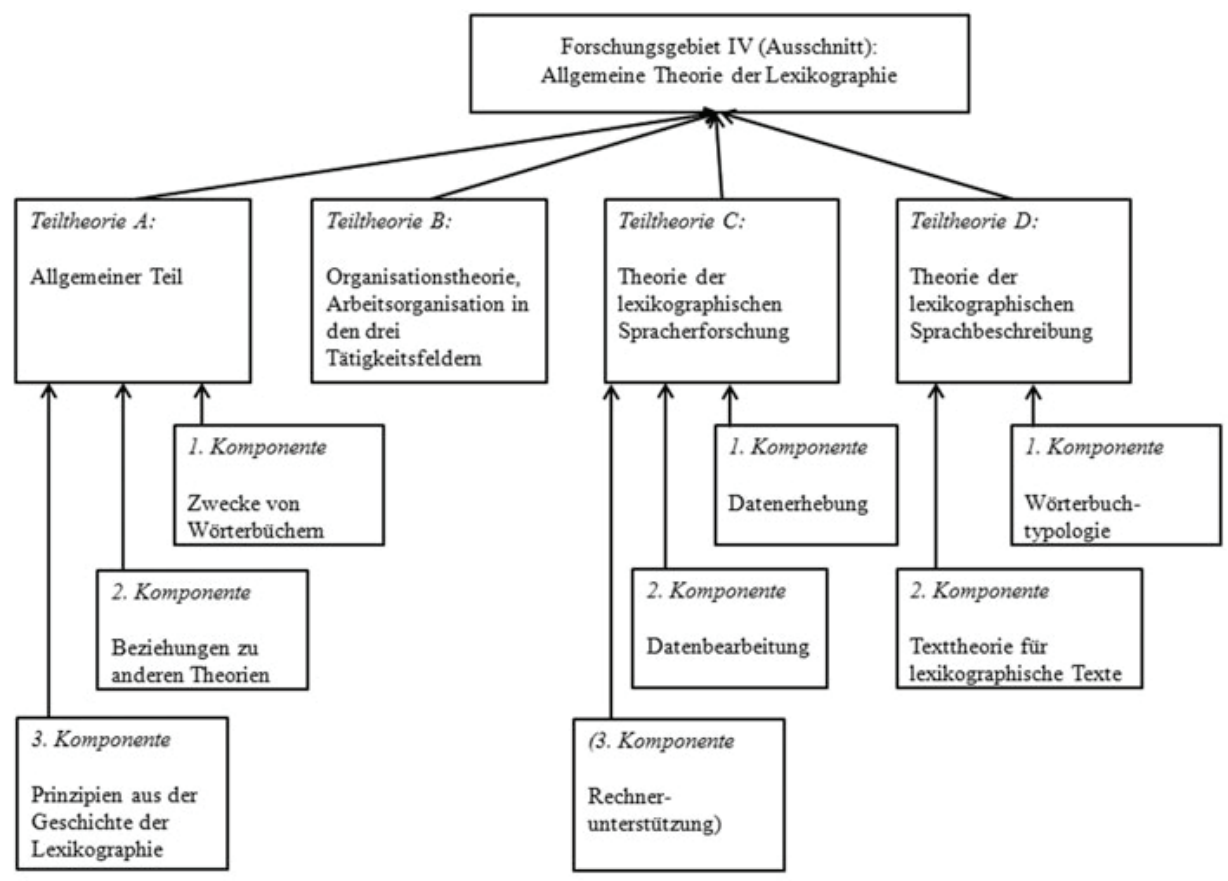

Abb. 3: Strukturierung einer allgemeinen Theorie der Lexikographie (cf. Wiegand 1984, 560)

Eine lexikografische Methode definiert Wiegand als „eine geordnet anzuwendende Menge von Vorschriften, mit denen festgelegt wird, wie etwas lexikographisch $\mathrm{zu}$ bearbeiten ist“" $(1984,568){ }^{14}$

einer Korpusanalyse getroffen werden. Außerdem diskutieren sie, wie groß ein Korpus sein muss, um als Datenbasis für ein Wörterbuch zu dienen, und nennen 50-100 Millionen laufende Textwörter als Grundlage für ein allgemeinsprachliches Wörterbuch mit 50.000 bis 100.000 Stichwörtern (cf. Bergenholtz/Mugdan 1990, 1620f.)

14 In diesem Zusammenhang grenzt Wiegand den Begriff „Methode“ auch vom Terminus „Prinzip“ ab: „Ein lexikographisches Prinzip legt fest, daß etwas Bestimmtes gemacht werden muss“ (Wiegand 1984, 568). 
Wiegand erwähnt neben den lexikografischen Datenerhebungsmethoden, die er nicht weiter ausführt, auch die Methoden der Datenbeschreibung, die er in offene und geschlossene Methoden untergliedert (cf. Wiegand 1984, 568f.). Die geschlossenen Beschreibungsmethoden bestehen aus einer vollständig determinierten Liste von Vorschriften, die dem „Bearbeiter keinen oder nur einen geringen Formulierungsspielraum“ (Wiegand 1984, 568) geben. Umfassen diese Methoden auch Vorschriften zur Abfolge der Textsegmente, dann führt ihre Anwendung „zu partiell oder vollständig standardisierten Wörterbuchartikeln mit relativ genau voraussagbarem Textverdichtungsgrad“ (Wiegand 1984, 569). In Printwörterbüchern werden die Angaben zur Aussprache und zur Grammatik überwiegend mit geschlossenen Beschreibungsmethoden erstellt. Demgegenüber ist bei den offenen Beschreibungsmethoden die Liste der Vorschriften nicht vollständig determiniert, sondern offen, wodurch der Bearbeiter größere Freiheiten beim Formulieren hat, was „zu nichtstandardisierten, z.T. narrativen lexikographischen Texten“ (Wiegand 1984, 569) führt. Die Bedeutungserläuterungen gehören zu den Angaben, die durch offene Methoden der Datenbeschreibung entstehen. Offene und geschlossene Beschreibungsmethoden kommen auch kombiniert vor. Anschließend beschreibt Wiegand am Beispiel der etymologischen, der bedeutungsgeschichtlichen und der historischen Autorenwörterbücher die Auswahlmethoden für Lemmata und einige Methoden zur Beschreibung der Bedeutung(sgeschichte). Für die historischen Autorenwörterbücher führt er als Methoden der Bedeutungsbeschreibung an: die Nennung eines Wortäquivalents, die Kumulation von Wortäquivalenten, die kurze oder lange lexikalische Paraphrase, die relativ ausführliche Sachbeschreibung und Hinweise zur Bedeutungsentwicklung.

Reichmann (2012, 218ff.) beschreibt Arbeitsgänge zur Gewinnung von Bedeutung in historischen Wörterbüchern und unterscheidet dabei zwischen dem Orientierungsgang, der Beleginterpretation und dem Sortierungsgang. ${ }^{15} \mathrm{Im}$ Orientierungsgang liest der Lexikograf alle vorhandenen Belege durch und erfasst überblicksartig ihren Inhalt. Der Schritt der Beleginterpretation dient dazu, einzelbelegspezifische Bedeutungshypothesen aufzustellen, wobei dies auch parallel zum Orientierungsgang geschehen kann. Im Sortierungsgang werden schließlich die einzelbelegspezifischen Bedeutungshypothesen gegliedert - ähnliche Belege werden zusammengefasst, unterschiedliche voneinander abgegrenzt, bis ein Gliederungsentwurf für den Wörterbuchartikel steht. Exemplarisch stellt Reichmann (2012, 224ff.) Möglichkeiten vor, wie Lexikografen zu einzelnen Bedeutungserläuterungen kommen können: 1 . Übernahme eines (zum Ausdruck der Behandlungssprache synonymen) Belegausdrucks in die Paraphrase und deren konstruktive Verarbeitung, 2. Übernahme eines übersetzten Belegausdrucks in die Paraphrase, 3. Übernahme eines Belegausdrucks, der

15 Obwohl sich Reichmanns Darstellung nicht an synchronen Wörterbüchern orientiert, wird sie hier kurz vorgestellt, weil sie sich allein auf Methoden der Erstellung von semantischen Paraphrasen bezieht. 
einen paradigmatischen oder etymologischen Bezug zum behandlungssprachlichen Ausdruck aufweist oder sich aus einem ähnlichen Frame, einer Isotopiekette, einer syntaktischen Folge bzw. aus Assoziationen ergibt, 4. Übernahme eines eigenen oder in der Literaur gelesenen Ausdrucks in die Paraphrase, 5. abstrahierte Ausdrücke aus den Belegen und deren gegliederte Darstellung (z.B. 2a, b, ...), 6. additive Reihung der Ausdrücke, 7. Ineinandergreifen intensionaler und extensionaler Formulierungen (cf. Abschnitt 3.3).

\subsection{Typen von Bedeutungsparaphrasenangaben}

Wie Bedeutungsparaphrasenangaben erstellt werden, hängt nicht zuletzt davon ab, um welchen Typ von Bedeutungserläuterung es sich handelt. Das betrifft sowohl den Aspekt der Datenerhebung als auch den Akt des Formulierens, denn der Paraphrasentyp beeinflusst einerseits die inhaltliche Seite der Paraphrase, andererseits auch deren sprachliche Ausgestaltung.

Es gibt zahlreiche Versuche, die einzelnen Arten von Bedeutungserläuterungen zu benennen und zu typologisieren. Die Ansätze fallen in ihrem Umfang und Inhalt recht unterschiedlich aus und beziehen sich auf verschiedene Arten von Wörterbüchern. In monolingualen, allgemeinsprachlichen Wörterbüchern der Gegenwartssprache spielen vor allem die folgenden Paraphrasentypen eine Rolle, wie sie bei Schlaefer $(2009,95 f$.$) aufgeführt werden. { }^{16}$

1. Die logische Bedeutungserläuterung orientiert sich am aristotelischem Vorbild, es wird ein Oberbegriff (Genus proximum) genannt, der durch charakteristische Unterschiede (Differentia specifica) näher spezifiziert wird, z.B.: April 'vierter Monat des Jahres' (Duden online). ${ }^{17}$

2. In der paradigmatischen Bedeutungserläuterung werden Synonyme (seltener Antonyme) für die Beschreibung der Wortbedeutung herangezogen, z.B.: lebhaft 'deutlich, klar, genau'18 (Duden online). ${ }^{19}$

3. Die morpho-semantische Bedeutungserläuterung findet bei Wortbildungsprodukten Verwendung, sie paraphrasiert „das syntagma-interne Bedeutungsver-

16 Herbst/Klotz (2003, 33ff.) bezeichnen die verschiedenen Typen von Bedeutungserläuterungen als Methoden.

17 Schmidt (1981, 46ff. und 62f.) bezeichnet diese Art Bedeutungserläuterung als „Sachdefinition“ bzw. „Bedeutungsdefinition“, bei Bosque (1982, 106f.) wird sie als hyperonymische Definition beschrieben. Atkins/Rundell (2008, 414ff.) führen sie unter der Bezeichnung „traditional model“. Weiterhin üblich ist die Benennung „,analytical definition“ (Geeraerts 2013, 489) oder ,analytic definition“ (Ayto 1983, 89).

18 Bei polysemen Wörtern wird nur die Lesart zitiert, deren semantische Paraphrase dem vorgestellten Typ entspricht.

$19 \mathrm{Zu}$ synonymischen Bedeutungserläuterungen cf. auch Svensén (2009, 214ff.) und Atkins/Rundell (2008, 420ff.). 
hältnis der beiden unmittelbaren Konstituenten“ (Schlaefer 2009, 95) und kann mit der Wortbildungsbedeutung gleichgesetzt werden, z.B.: Geburtstagsgeschenk 'Geschenk zum Geburtstag' (Duden online).

4. Die Negations-/Ausschlussparaphrase verwendet für die Bedeutungserläuterung Verneinungen des Gegenteils, z.B.: locker 'nicht straff [gespannt], nicht starr; nicht fest' (Duden online).

5. Die Funktionsparaphrase wird vorwiegend bei den synsemantischen Wortarten benutzt, sie benennt ,grammatische, semantische oder pragmatische Funktionen“ (Schlaefer 2009, 96), z.B.: was 'bezeichnet in Relativsätzen dasjenige, worüber im Relativsatz etwas ausgesagt ist' (Duden online).

6. Bei der Einwortparaphrase besteht die Bedeutungserläuterung lediglich aus einem einzigen Wort), z.B.: Zündholz 'Streichholz' (Duden online).

7. Die Rektionsparaphrase wird vor allem bei Verben eingesetzt und zeigt in ihrer Struktur bereits die Rektion), z.B.: helfen 'jemandem durch tatkräftiges Eingreifen, durch Handreichungen oder körperliche Hilfestellung, durch irgendwelche Mittel oder den Einsatz seiner Persönlichkeit ermöglichen, [schneller und leichter] ein bestimmtes Ziel zu erreichen; jemandem bei etwas behilflich sein, Hilfe leisten' (Duden online).

8. Die metalinguistische Bedeutungserläuterung „weist Relationsprädikate auf, die eine metalinguistische Aussage über die Funktion des Stichwortes enthalten“ (Schlaefer 2009, 96), z.B.: Raffel '(landschaftlich salopp abwertend) großer, als hässlich empfundener Mund' (Duden online). ${ }^{20}$

Neben diesen acht Typen von Bedeutungsparaphrasenangaben verzeichnet Schlaefer (2009, 95f.) noch die taxonomische Bedeutungserläuterung, die allerdings stärker in Fachwörterbüchern Verwendung findet, da sie begriffliche Taxonomien voraussetzt, sowie die ostensive Bedeutungserläuterung, bei der eine Abbildung die Funktion der semantischen Paraphrase übernimmt. ${ }^{21}$ Schmidt $(1981,54)$ führt für fachsprachliche Begriffe, „für deren inhaltliche Bestimmung besonders hohe Genauigkeit angestrebt wird“, noch die „Begriffsdefinition“ auf, die allerdings ebenfalls überwiegend in Fachwörterbüchern zu finden ist.

Anstelle solcher Bemühungen, Typen semantischer Paraphrasen zu klassifizieren, setzen einige Wissenschaftler gegensätzliche Begriffspaare ein, die unterschiedliche Ausprägungen von Bedeutungsparaphrasenangaben beschreiben. Häufig steht steht dabei die formale Gestaltung der Paraphrase im Zentrum. Am Beispiel historischer Wörterbücher stellt Reichmann (2012, 286ff.) ausführlich die folgenden Paare gegenüber:

1. Einfache und komplexe Bedeutungserläuterungen: Die einfache Erläuterung besteht aus einem oder einigen wenigen Synonymen oder einer kurzen Wortgruppe. Die komplexe Bedeutungserläuterung nutzt „längere Reihungen von 
Synonymen und ausführliche, syntaktisch vielfach gestaffelte Wortgruppen“ (Reichmann 2012, 287). Diese beiden Formen korrespondieren mit den Erläuterungen für Rezeptions- und für Produktionszwecke (cf. Atkins/Rundell 2008, 407ff.). Will der Nutzer zum Beispiel beim Lesen eines Textes ein ihm unbekanntes Wort verstehen, genügen ihm dafür wenige Informationen. Um ein Wort in einer sprachproduktiven Aufgabe aktiv zu verwenden, sind hingegen viel mehr Informationen nötig, unter anderem zu semantischen und pragmatischen Eigenschaften.

2. Synonymische und phrastische Bedeutungserläuterungen: Die Erläuterungen durch Synonyme oder durch eine Wortgruppe bilden keinen Gegensatz, sie können auch kombiniert vorkommen.

3. Kompakte und diffuse Bedeutungserläuterungen: Kompakte Erläuterungen stellen das Bedeutungsspektrum am Stück vor, bei einer diffusen Bedeutungsparaphrasenangabe wird diese immer wieder durch Belege o. Ä. unterbrochen. Diffuse Bedeutungserläuterungen finden sich allerdings typischerweise in historischen Wörterbüchern.

Als weitere Begriffspaare dieser Art können gelten:

4. Intensionale und extensionale Bedeutungserläuterungen (cf. Svensén 2009, 218ff.; Geeraerts 2013, 489): Die intensionale Bedeutungserläuterung nutzt das Verfahren der logischen Definition mit Genus proximum und Differentia specifica. Die seltener vorkommenden extensionalen Erläuterungen finden bei Kollektiva Verwendung, sie zählen Hyponyme zum beschriebenen Lexem auf und können dadurch auch als eine Form der paradigmatischen Bedeutungserläuterung gelten, z.B.: Eltern 'Vater und Mutter' (Duden online).

5. Bedeutungserläuterungen in Wortgruppen- oder Satzform (cf. Hanks 1987; Svensén 2009, 235ff.; Atkins/Rundell 2008, 441ff.): Traditionell wurden Bedeutungsparaphrasenangaben in Printwörterbüchern in Form einer Wortgruppe abgefasst, als Begründung hierfür wurden häufig Platzbeschränkungen angegeben. Ende der Achtzigerjahre brach Collins mit dem COBUILD-Wörterbuch für nichtmuttersprachliche Englischlerner mit dieser Tradition. Die Erläuterungen in vollständigen Sätzen sollten stärker an alltägliche Sprache erinnern und so die Verständlichkeit erhöhen.

Welcher Paraphrasentyp im Einzelfall gewählt wird, hängt von einer Vielzahl von Faktoren ab. Die erste Gruppe von Aspekten betrifft dabei das Wörterbuch an sich, in dem die Bedeutungsparaphrasenangabe stehen soll. So sind je nach Wörterbuchtyp unterschiedliche Arten von Bedeutungserläuterungen üblich, die auch in Umfang und Tiefe der Information variieren. ${ }^{22}$ In direktem Zusammenhang damit steht die Zielgruppe des Wörterbuchs. Handelt es sich beispielsweise um ein historisches Wörterbuch, das sich überwiegend an Spezialisten richtet, ist eher mit komplexen, phrastischen Erläuterungen zu rechnen als in einem Rechtschreibwörterbuch, das einen

22 Bedeutungsparaphrasen finden sich nur in einem Teil der Wörterbücher, da mehrsprachige Wörterbücher keine semantischen Paraphrasen angeben, sondern Äquivalente in der Zielsprache bzw. den Zielsprachen. 
allgemeinen Nutzerkreis anvisiert: „Ein an Experten adressiertes Wörterbuch wird in den Definitionen einen differenzierten Wissensstandard mit umfangreicher Berücksichtigung der maßgeblichen Sachzusammenhänge bieten, während ein an Laien adressiertes Wörterbuch aus didaktischen Gründen benutzerspezifische Zurücknahmen und Vereinfachungen im Wissensstand zu berücksichtigen hat.“ (Schlaefer 2009, 95). Auch im Bereich der didaktischen Wörterbücher wirkt sich der vermutete Wissensstand der fremdsprachigen oder muttersprachlichen Lerner auf die inhaltliche Komplexität bzw. Einfachheit der semantischen Paraphrase aus. Lernerwörterbücher für nichtmuttersprachliche Nutzer oder Wörterbücher für muttersprachliche Lerner sollten in ihren Bedeutungsparaphrasenangaben neben der inhaltlichen Seite bei der formalen Gestaltung der Paraphrasen noch zusätzliche Aspekte stärker berücksichtigen, etwa, dass das verwendete Beschreibungsvokabular nicht zu umfangreich und schwierig ist oder dass die syntaktischen Strukturen nicht zu komplex sind. Nicht zuletzt beeinflussen auch die Regelungen, die für das konkrete Wörterbuch getroffen werden, in welcher Form die Bedeutungserläuterung erstellt wird. So legen Redaktionshandbücher bzw. „Style Guides“ beispielsweise fest, ob in ganzen Sätzen oder in Wortgruppen definiert wird.

Die zweite Gruppe von Einflussfaktoren hängt mit den speziellen Charakteristika des zu beschreibenden Lexems zusammen. Die typische logische Bedeutungserläuterung lässt sich lediglich auf Vertreter der Autosemantika anwenden, bei den synsemantischen Wortarten muss auf eine Funktionsparaphrase zurückgegriffen werden. Auch die Wortart des Lexems bzw. seine grammatischen Eigenschaften spielen eine Rolle, wenn es um die Wahl eines angemessenen Paraphrasentyps geht. Verben können effektiv mithilfe einer Rektionsparaphrase umschrieben werden, die logische Bedeutungserläuterung findet sich häufig bei Nomen. Weiterhin kommt es auf die semantische Klasse des Lexems an - so bietet sich eine extensionale Bedeutungsparaphrase lediglich bei Kollektiva an, deren Hyponyme eine nicht zu umfangreiche Gruppe bilden. Die paradigmatische Struktur des Lexems ist ebenfalls entscheidend, wenn der Lexikograf eine Bedeutungserläuterung mittels Synonymen einsetzen möchte, denn hierfür ist eine ausreichende paradigmatische Vernetztheit des Lexems nötig. Um eine metalinguistische Bedeutungserläuterung verwenden zu können, muss das Lexem Konnotationen aufweisen. Die Gebildetheit des Lemmas, also eine komplexe morphologische Struktur bei gleichzeitiger Motiviertheit, bildet die Voraussetzung dafür, dass es mithilfe einer morpho-semantischen Paraphrase umschrieben werden kann.

Ein letzter Punkt, der für die Wahl des Paraphrasentyps eine Rolle spielt und der keinesfalls unterschätzt werden sollte, ist der Lexikograf. Sein persönlicher Stil und seine Formulierungsvorlieben beeinflussen auch die Ausgestaltung der Bedeutungsparaphrasen in ihrer Komplexität bzw. Einfachheit. Letztendlich bestimmt der Lexikograf mit seiner Entscheidung für einen Paraphrasentyp auch, welche Art semantischer Informationen der Nutzer in der Bedeutungserläuterung erhält, wie auch Geeraerts $(2013,490)$ feststellt: „some definitional techniques are most suited 
for a specific type of semantic information“. Selbst ausführliche redaktionelle Vorgaben und umfangreiche Korrekturgänge bei der Erstellung eines Wörterbuchs können oft nichts daran ändern, dass semantische Paraphrasen die individuelle Handschrift ihres lexikografischen Autors tragen.

\section{Das Beispiel elexiko - Vorgehen beim Erstellen der semantischen Paraphrase}

Seit mehr als zehn Jahren wird am Institut für Deutsche Sprache in Mannheim das Onlinewörterbuch elexiko (kurz für: elektronisches, lexikalisch-lexikologisches und korpusbasiertes Informationssystem) erstellt. ${ }^{23}$ Dieses einsprachige Wörterbuch zum Gegenwartsdeutschen gehört zum Wörterbuchportal OWID. ${ }^{24}$ Die Wörterbuchartikel für elexiko werden mithilfe der korpuslinguistischen und statistischen Methoden erarbeitet, die COSMAS II und die Kookkurrenzdatenbank CCDB zur Verfügung stellen. ${ }^{25}$ Die Textgrundlage bildet dabei das bis Ende 2013 regelmäßig aktualisierte elexiko-Korpus, das mit seinen rund 2,7 Milliarden Textwörtern aus deutschen, österreichischen sowie Schweizer Zeitungs- und Zeitschriftentexten zusammengestellt ist.

Die Stichwortliste von elexiko umfasst rund 300.000 Einträge, die in unterschiedlicher Tiefe bearbeitet werden. Alle Lemmata werden mit breit angelegten, einfachen Informationen (z.B. zur Orthografie oder Worttrennung) sowie drei automatisch ausgewählten Belegen ausgestattet. Begrenzte Wortschatzbereiche, aktuell das „Lexikon zum öffentlichen Sprachgebrauch“, erhalten umfangreiche Informationen in einer sehr komplexen Artikelstruktur. Solche redaktionell bearbeiteten Wortartikel enthalten Angaben zur Bedeutung, Verwendung, Grammatik, Rechtschreibung und Wortbildung. Außerdem bieten gesprochensprachliche Belege die Möglichkeit, die Aussprache und Betonung des Stichwortes im Satzzusammenhang zu hören. Wo es sinnvoll ist, begleiten ein bis drei Illustrationen in einer oder mehreren Lesarten eines

23 Die ursprüngliche theoretische Konzeption von elexiko wird in Haß (2005a) vorgestellt. Zur praktischen Umsetzung nach einigen Jahren Artikelarbeit cf. Klosa (2011b). Einen kurzen Einblick in das Projekt bieten auch die Internetseiten unter http://www.owid.de/wb/elexiko/start.html. Diese drei Quellen behandeln auch einzelne Aspekte wie die Stichwortliste oder das elexiko-Korpus ausführlicher.

24 Einzelheiten dazu, wie OWID konzipiert wurde und wie es sich entwickelt hat, werden in Müller-Spitzer (2007; 2010), in Engelberg/Klosa/Müller-Spitzer (2009) sowie auf den Internetseiten des Projekts unter http://www.owid.de dargelegt.

25 Details zu COSMAS II und zur Kookkurrenzdatenbank CCDB finden sich auf den jeweiligen Internetseiten der Projekte unter https://cosmas2.ids-mannheim.de/cosmas2-web/ bzw. http://corpora. ids-mannheim.de/ccdb/. 
Stichwortes die Bedeutungserläuterung. Der Ausbau von elexiko erfolgt also nicht von A bis Z, sondern modular. ${ }^{26}$

Zur redaktionellen Arbeitsumgebung bei elexiko gehören vor allem die COSMAS-II-Schnittstelle als Verbindung zum elexiko-Korpus, der XML-Editor zum Verfassen der Wörterbuchartikel, die Schnittstelle zur ORACLE-Datenbank sowie der Verweismanager, der bei den Verweisen stark zur Konsistenzsicherung beiträgt (cf. Klosa 2011a, 14ff.). Daneben steht den Lexikografen ein in die DTD-Dokumentation integriertes Redaktionshandbuch zur Verfügung, in dem ,alle Entscheidungen zu Formulierungen (z.B. der Satzbaupläne bei Verben), Richtlinien für die Verwendung von Hinweisen oder Kommentaren, Leitlinien für die Auswahl von Belegen etc. festgehalten werden, um die Arbeit an neuen Wortartikeln wie die Einarbeitung neuer Kollegen zu erleichtern“ (Klosa 2011a, 15). Das Redaktionshandbuch betrifft also alle lexikografischen Angabebereiche. Es kann kontextsensitiv aus dem XML-Editor geöffnet werden.

Doch wie entsteht eine semantische Paraphrase bei elexiko nun genau? Welche Arbeitsschritte bewerkstelligt der Lexikograf dabei? Dies soll im Folgenden anhand des Beispiels Freude beschrieben werden. ${ }^{27}$ An den passenden Stellen werden dabei die theoretischen Überlegungen dargelegt, die hinter elexiko stehen.

Den Ausgangspunkt für die konkrete lexikografische Arbeit bildet die Erhebung der Daten, die später Eingang in den Wortartikel finden sollen. Wie im Abschnitt 2.3 beschrieben, werden als Methoden der Datenerhebung bei Bergenholtz/Mugdan (1990) die Auswertung vorliegender Beschreibungen, die Introspektion, die Informantenbefragung, die Auswertung einer Belegsammlung und die Auswertung eines Korpus genannt (cf. Bergenholtz/Mugdan 1990, 1612). Bei elexiko als korpusbasiert erarbeitetem Wörterbuch dominiert die Methode der Korpusauswertung, aber begleitend spielen natürlich auch die Auswertung bereits vorliegender Beschreibungen und die Introspektion des Lexikografen eine Rolle. ${ }^{28}$ Ausdruck der Korpusauswertung ist die Liste der Kookkurrenzen für das betreffende Stichwort, die mittels COSMAS II erstellt wird und für die alle Vorkommen des Stichwortes im elexiko-Korpus analysiert werden. Abb. 4 zeigt den Beginn dieser Kookkurrenzliste für das Beispiel Freude.

26 Klosa (2011a) beschreibt den modularen Ausbau von elexiko und das „Lexikon zum öffentlichen Sprachgebrauch“ genauer.

27 Natürlich können hier nicht sämtliche Arbeitsabläufe erschöpfend dargestellt werden. Da an dieser Stelle der Fokus auf dem methodischen Vorgehen beim Erstellen einer Bedeutungsparaphrase liegt, werden bestimmte Schritte ausgeblendet, die in der konkreten lexikografischen Tätigkeit zwar nötig sind, aber nicht direkt mit dem Verfassen der semantischen Paraphrase zu tun haben. Solche Schritte sind beispielsweise das Auschecken des Wortartikels aus der Datenbank oder das Öffnen des Wortartikels in einem XML-Editor.

28 Bergenholtz/Mugdan (1990, 1612f.) bemängeln, dass bei vielen Wörterbuchprojekten nicht offengelegt wird, welche vorhandenen Quellen auf welche Weise genutzt werden. Zum Umgang mit Quellen in elexiko cf. Klosa (2013). 

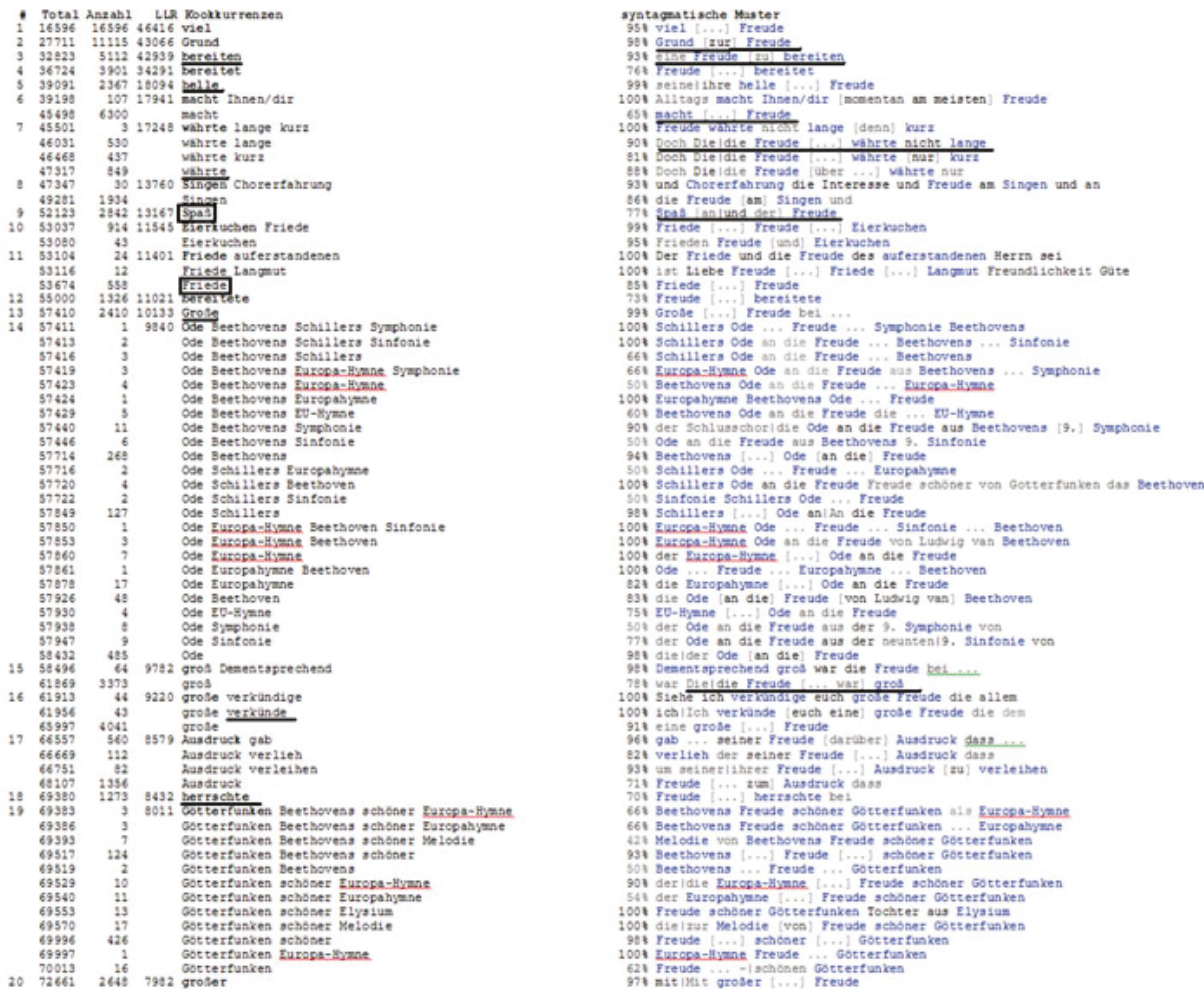

Abb. 4: Ergebnis der Kookkurrenzanalyse für das Lemma Freude im elexiko-Korpus

Diese Liste wertet der Lexikograf im Laufe seiner Arbeit aus und gelangt so zu den Daten für die einzelnen Angabebereiche seines Wörterbuchartikels. ${ }^{29}$ Die Schritte der Datenerhebung und der Datenbeschreibung laufen an dieser Stelle parallel ab und sind mit dem Orientierungsgang, der Beleginterpretation und dem Sortierungsgang bei Reichmann (2012, cf. auch Abschnitt 3.2) vergleichbar. Die fünfte Spalte (Kookkurrenzen) dient dabei vor allem als Grundlage für den Angabebereich „Kollokationen“, der zeigt, „wie sich um das Stichwort als Zentrum die Mitspieler als Netz zur semantischen Umgebung auf syntagmatischer Ebene anordnen, wobei zugleich syntaktische Informationen vermittelt werden“ (Klosa/Storjohann 2011, 49). Die Kollokationen werden in Sets aus Fragen und jeweils passenden Antworten sortiert. Aus den ersten zwanzig Kookkurrenzen ergeben sich diese Daten zu den syntagmatischen Positionen und ihren Füllungen (in Spalte 5 von Abb. 4 unterstrichen): „Wie ist Freude? - groß, hell“, „Was macht man mit Freude? - bereiten, verkünden“, „Was macht Freude? - 
herrschen, währen“, „Wobei bzw. woran hat man Freude? - Singen“. Auf die Frage „Wer empfindet Freude?“ liefern erst spätere Kookkurrenzen eine Antwort. Aus der Kookkurrenzen-Spalte ergeben sich auch Hinweise für einige paradigmatische Partner (in Spalte 5 von Abb. 4 eingerahmt): Frieden und Spaß sind inkompatible Ausdrücke $\mathrm{zu}$ Freude. Aus der sechsten und letzten Spalte (syntagmatische Muster) zieht der Lexikograf die Daten für den Angabebereich „Konstruktionen“ (in Spalte 6 von Abb. 4 unterstrichen) - „prototypische, usuelle phrasale Muster mit konkreten korpusbasierten Lexemelementen“ (Storjohann 2005b, 236). Die Basis für solche typischen Verwendungen sind im Beispiel Freude unter anderem die Formulierungen „Grund zur Freude“, „eine Freude bereiten“, „macht Freude“, „,doch die Freude währte nicht lange“, „Spaß an der Freude“ und „die Freude war groß“. Die Spalten mit den Kookkurrenzen und den syntagmatischen Mustern bietet auch Anhaltspunkte für die syntaktischen Anschlüsse des Lemmas (Freude an jemandem/etwas, Freude bei etwas, Freude mit jemandem/etwas, Freude über jemandem/etwas, Freude, dass [...]), die im Angabebereich „Grammatik“ aufgeführt werden.

Die Arbeit an der Kookkurrenzliste vermittelt dem Lexikografen also ein erstes Bild von der Bedeutung und Verwendung des Stichwortes. ${ }^{30}$ Mit den Kollokationen erhält er semantische Informationen zur Argumentstruktur des Lemmas, die Konstruktionen illustrieren ihm, wie das Stichwort in seiner jeweiligen Lesart oder Lesartenspezifizierung im Satz oder in der Phrase eingebettet ist, und mit der Angabe der sinnverwandten Wörter werden ihm die paradigmatischen Beziehungen des Lemmas verdeutlicht. Zudem hat er zu diesem Zeitpunkt bereits zahlreiche Korpusbelege des Lemmas gelesen, unter anderem, um unklare Kollokationen zu verstehen und eventuell zu belegen, um Füllungen für Konstruktionen zu finden oder um die Relationen zu den sinnverwandten Partnerwörtern zu belegen.

An dieser Stelle der Artikelarbeit kümmern sich die meisten Lexikografen bei elexiko um die Vervollständigung des Angabebereichs „Grammatik“ mit den Daten zur Wortart, Flexion, Satzstellung und Valenz. In einem nächsten Schritt wird überprüft, ob das Lemma Gebrauchsbesonderheiten aufweist, die dann in dem entsprechenden Angabebereich beschrieben werden. Im Normalfall verfasst der Lexikograf erst danach die semantische Paraphrase, denn das Wissen über alle anderen Angabebereiche bildet die Voraussetzung für eine passende Bedeutungserläuterung. So sieht es auch das Redaktionshandbuch vor, das festlegt, die semantische Paraphrase erst $\mathrm{zu}$ formulieren, nachdem eine detaillierte semantische Analyse erfolgt ist, also die

30 Die Analyse der Kookkurrenzen zeigt auch, in wie viele Lesarten und Lesartenspezifizierungen sich das Lemma aufgliedert. Bei einem polysemen Lemma müssen die einzelnen Kollokationen, Konstruktionen und paradigmatischen Partner auf die unterschiedlichen Lesarten und Lesartenspezifizierungen verteilt werden. Je nach semantischer Nähe fällt das schwerer oder leichter. Aus Gründen der Einfachheit wurde an dieser Stelle mit Freude ein monosemes Lemma zur Veranschaulichung gewählt. 
lesarten- und kontexttypenspezifischen Inhalte auf Merkmale und Funktionen hin untersucht worden sind. Die Bedeutungserläuterung entsteht bei den elexiko-Redakteuren also mit Absicht am Ende der Arbeit an einem Wortartikel: „Auf diese Weise kann die semantische Paraphrase bewusster auf alle anderen Angabebereiche abgestimmt und mit diesen verzahnt werden, sodass die Bedeutungserläuterung schließlich ein Kondensat vieler Erkenntnisse anderer Angabebereiche darstellt.“ (Töpel 2011, 46).

Die Erstellung der semantischen Paraphrasen geschieht bei elexiko nach festgelegten Prinzipien, die im Redaktionshandbuch dokumentiert sind und die beim Schreiben des Wörterbuchartikels jederzeit eingesehen werden können. ${ }^{31} \mathrm{Zu}$ ihnen gehören das Ganzsatzmodell, die Orientierung an der satzfunktionalen Klasse der Lesart, die Integration der Kurzetikettierung sowie die Angabe konkreter Beispiele. ${ }^{32}$ Die lexikografischen Methoden, mittels derer diese Prinzipien umgesetzt werden, sind grundsätzlich offen, die Bedeutungserläuterung bei elexiko ist demzufolge ein nichtstandardisiertes Textsegment (cf. Abschnitt 3.2).

Das Formulieren in ganzen Sätzen lässt sich methodisch knapp zusammenfassen - die Bedeutungsparaphrase muss den formalen Kriterien eines Satzes genügen, also zumindest ein finites Verb und die nötigen Verbkomplemente enthalten (cf. grammis)..$^{33}$

Das aktuell in elexiko bearbeitete Modul, das „Lexikon zum öffentlichen Sprachgebrauch“, besteht zum großen Teil aus Autosemantika, weshalb sich die Wörterbucharbeit auf diese Wortarten konzentriert. Die synsemantischen Wortarten sind - mit Ausnahme der Artikelwörter und Pronomen - momentan nicht systematisch beschrieben. Jedoch gibt es auch für Junktoren, Partikel, Präpositionen und interakive Einheiten bereits ausgearbeitete Wortartikel, vor allem, weil einige primär autosemantische Lemmata auch über synsemantische Lesarten verfügen. In elexiko erhalten die Synsemantika als Beschreibung ihrer Verwendung eine syntaktische Funktionsregel. Bei den autosemantischen Lemmata bzw. Lesarten richtet sich die semantische Paraphrase nach der satzfunktionalen Klasse des entsprechenden Lemmas bzw. der entsprechenden Lesart und ist demzufolge auf die Eigenschaften der Satzfunktion

31 Die theoretische Konzeption zur Bedeutungserläuterung in elexiko präsentiert Storjohann (2005a), ihre Umsetzung in der lexikografischen Praxis und die Beurteilung durch die Lexikografen beschreibt Töpel (2011).

32 Die Kurzetikettierung bezeichnet in elexiko die einzelne Lesart eines Stichwortes, beispielsweise Folge, Lesart 'Auswirkung' oder 'fortlaufende Reihe'. Sie ersetzt die in der Printlexikografie übliche Nummerierung der Lesarten.

33 Storjohann (2005a, 190) stellt die Vorteile des Ganzsatzmodells für elexiko aus konzeptioneller Perspektive dar. Zur Anwendung der Satzparaphrasen im COBUILD-Wörterbuch sowie zur grundsätzlichen Diskussion über Bedeutungserläuterungen in ganzen Sätzen cf. beispielsweise Rundell (2006). 
abgestimmt. ${ }^{34}$ Für jede Klasse (Prädikator, Quantor, Referenzwort) und ihre Untertypen gibt es bestimmte Beschreibungsmuster, die „ein Orientierungsinstrumentarium zum Formulieren der Bedeutungserläuterung darstellen“ (Storjohann 2005a, 186). Auch diese sind im Redaktionshandbuch festgehalten. In Abhängigkeit von semantischen oder grammatischen Kriterien sowie vom Referenzbereich sortiert der Lexikograf das zu beschreibende Lemma bzw. die zu beschreibende Lesart also zunächst in eine satzfunktionale Klasse und dann in eine Untergruppe ein. Anschließend kann er sich bei der Erstellung der Paraphrase an den Formulierungsvorschlägen orientieren. Es folgt eine Übersicht über die satzfunktionalen Klassen und ihre Untertypen mit den zugehörigen Beschreibungsmustern und einem Beispiel:

1. Prädikator:

a. Gattungsprädikator:

- Individuativum: „Mit ... bezeichnet man/bezeichnen Sprecher/bezieht man sich/beziehen sich Sprecher auf ..., der/die/das ...“ oder „Mit ... wird/werden ... bezeichnet/gekennzeichnet.“ oder „Mit ... wird/werden auf ... Bezug genommen.“

- Beispiel Brücke, Lesart 'Bauwerk': 'Mit Brücke bezeichnet man ein Bauwerk, das eine Verbindung zwischen zwei Orten herstellt, die durch einen Fluss, ein Tal o.̈̈. voneinander getrennt sind.' (elexiko)

- Kollektivum: „Mit ... bezeichnet man/bezeichnen Sprecher/bezieht man sich/beziehen sich Sprecher auf die Gesamtheit von ...“ oder „Mit ... wird die Gesamtheit von ... bezeichnet“ oder „... bezeichnet eine Gesamtheit von ...“

- Beispiel Publikum, Lesart 'Zuschauer': 'Mit Publikum bezeichnet man die Gesamtheit aller Zuhörer oder Zuschauer eines Ereignisses (z.B. einer kulturellen Veranstaltung oder einer Sportveranstaltung).' (elexiko)

- Kontinuativum: „Mit ... bezeichnet man/bezeichnen Sprecher/bezieht man sich/beziehen sich Sprecher auf eine Substanz/einen Stoff/eine Masse von ...“ oder „... bezeichnet eine Substanz/einen Stoff/eine Masse von ...."

- Beispiel Papier, Lesart 'Material': 'Mit Papier bezeichnet man Material, das sehr dünn und häufig weiß ist und das vor allem zum Schreiben, Drucken und Verpacken verwendet wird.' (elexiko)

- Privativum: „Mit ... bezeichnet man/bezeichnen Sprecher/bezieht man sich/beziehen sich Sprecher auf das Fehlen/Nichtvorhandensein eines/ einer ..."

- Beispiel fehlen, Lesart 'nicht da sein': 'Mit fehlen bezeichnet man, dass ein Gegenstand, ein Sachverhalt oder eine Person(engruppe) nicht da ist, nicht vorhanden ist, nicht existiert.' (elexiko)

- Partitivum: „Mit ... bezeichnet man/bezeichnen Sprecher/bezieht man sich/beziehen sich Sprecher auf einen Untertyp/Teil ...“ 
Beispiel Hand, Lesart 'Teil des Arms': 'Mit Hand bezeichnet man beim Menschen oder Affen den unteren Teil des Arms, an dem sich die Finger befinden und der dazu dient, Gegenstände zu ergreifen oder festzuhalten.' (elexiko)

b. Handlungsprädikator: „Mit ... bezeichnet man/bezeichnen Sprecher/bezieht man sich/beziehen sich Sprecher auf eine Handlung, ...“ oder „Mit ... wird eine Handlung bezeichnet/gekennzeichnet, ...."

Beispiel tanzen, Lesart 'rhythmisch bewegen': 'Mit tanzen bezeichnet man eine Handlung, bei der sich eine Person(engruppe) rhythmisch zur Musik bewegt.' (elexiko)

c. Vorgangsprädikator: „Mit ... bezeichnet man/bezeichnen Sprecher/bezieht man sich/beziehen sich Sprecher auf einen Vorgang, ...“ oder „Mit ... wird ein Vorgang bezeichnet/gekennzeichnet, ...“

Beispiel steigen, Lesart ‘an Höhe gewinnen': 'Mit steigen wird ein Vorgang bezeichnet, bei dem sich ein Objekt nach oben bewegt und damit an Höhe gewinnt.' (elexiko)

d. Zustandsprädikator: „Mit ... bezeichnet man/bezeichnen Sprecher/bezieht man sich/beziehen sich Sprecher auf einen Zustand, ...“ oder „Mit ... wird ein Zustand bezeichnet/gekennzeichnet, ...“

Beispiel gesund, Lesart 'wohlauf': 'Mit gesund bezeichnet man den Zustand von Lebewesen, körperlich und geistig wohlauf sowie nicht von Krankheit betroffen zu sein. Sachverhalte (z.B. Lebensjahre oder die körperliche Entwicklung eines Menschen) werden mit gesund dahingehend charakterisiert, ohne Erkrankung zu verlaufen.' (elexiko)

e. Eigenschaftsprädikator: „Mit ... bezeichnet man/bezeichnen Sprecher die Eigenschaft ...“ oder „Mit ... wird ... die Eigenschaft zugewiesen/zugeschrieben, ...“

Beispiel vernünftig, Lesart 'sinnvoll': 'Mit vernünftig bezeichnet man die Eigenschaft von Sachverhalten (z.B. von Politik) und Handlungen (z.B. einer Antwort oder einer Erklärung), sinnvoll zu sein und von rationalem Verstand, Überlegtheit und Klugheit zu zeugen.' (elexiko)

f. Einstellungsprädikator: „Mit ... bewertet man/bewerten Sprecher ...“ oder „Mit ... wird ... als ... bewertet.“

Beispiel komisch, Lesart 'sonderbar': 'Mit komisch bewerten Sprecher eine Person(engruppe), einen Gegenstand oder einen Sachverhalt (z.B. ein Gefühl, eine Mischung) als sonderbar, eigenartig oder seltsam.' (elexiko)

2. Quantor: „... ist eine Bezeichnung für eine Maßeinheit/Menge, mit der ... gemessen wird.“ oder „... ist ein Ausdruck zum Messen von ...“ oder „Mit ... bezeichnet man/bezeichnen Sprecher eine Maßeinheit/Menge ...“ 35

Beispiel Hektar, Lesart 'Flächenmaß': 'Mit Hektar bezeichnet man eine Maßeinheit, mit der man die Ausdehnung von (z.B. landwirtschaftlichen) Flächen misst.' (elexiko) 
3. Referenzwort (Deiktika): „Mit ... wird auf ... verwiesen.“ oder „Mit ... verweist man auf ...."36

Beispiel draußen, Lesart 'im Freien': 'Mit draußen verweist man auf einen Ort, der sich im Freien befindet.' (elexiko)

Nachdem die satzfunktionale Klasse und die Untergruppe bestimmt sind, füllt der Lexikograf die Musterformulierung mit Inhalt. Dafür nutzt er das Wissen, das er beim Lesen der Korpusbelege und beim Verfassen der anderen Angabebereiche über das Lemma bzw. die Lesart gesammelt hat: Welche Kollokationen gibt es? Welche Konstruktionen sind typisch? Welche paradigmatischen Partnerwörter eignen sich beispielsweise als Hyperonym in der semantischen Paraphrase? Gibt es Besonderheiten im Gebrauch? Bestehen Valenzen?

Neben den Beschreibungsmustern, die in vielen Fällen bereits auf die Struktur der semantischen Paraphrase hindeuten, muss der Lexikograf noch darauf achten, die Kurzetikettierung sowie konkrete Beispiele in die Bedeutungserläuterung zu integrieren. So soll die Paraphrase stärker mit anderen Angabebereichen (wie den Kollokationen und den Konstruktionen) verknüpft werden, um einerseits beim Lesen einen Wiedererkennungseffekt beim Nutzer zu erreichen und andererseits die semantische Paraphrase expliziter zu gestalten. Die Wiederaufnahme der Etikettierung erfordert beim Formulieren der Bedeutungserläuterung teilweise viel verbales Geschick.

Am Beispiel von Freude (Lesart 'Glücksgefühl') soll die Entstehung der semantischen Paraphrase schrittweise nachvollzogen werden. Der Lexikograf hat die satzfunktionale Klasse (Gattungsprädikator, Individuativum) bestimmt und ein Beschreibungsmuster festgelegt: „Mit Freude bezeichnet man ...“. Von den möglichen Typen an Bedeutungserläuterungen entscheidet er sich für die logische Paraphrase mit Genus proximum und Differentia specifica. Aus dem Angabebereich der sinnverwandten Wörter sucht er sich ein geeignetes Hyperonym (Gefühl), das er mit Synonymen (Heiterkeit, Fröhlichkeit, Glück) näher spezifiziert: „Mit Freude bezeichnet man ein Gefühl der Heiterkeit, der Fröhlichkeit und des Glücks.“ Um den Nutzer beim Lesen der Bedeutungserläuterung zu lenken, werden in einem zweiten Satz typische Auslöser für Freude (Sachverhalte wie Musik oder Handlungen wie Tanzen) aus dem Angabebereich der Kollokationen aufgeführt (cf. Abb. 5).

Die bisherigen Ausführungen zu den Methoden, wie in elexiko Bedeutungsparaphrasen verfasst werden, und $\mathrm{zu}$ den Beschreibungsmustern für die einzelnen satzfunktionalen Klassen deuten bereits darauf hin, dass der Typ der logischen Paraphrase besonders häufig genutzt wird. Jedoch gibt es auch viele Beispiele, in denen andere Typen semantischer Paraphrasen verwendet werden. Gerade bei adjektivischen Stichwörtern bietet sich häufig eine paradigmatische Bedeutungserläuterung 


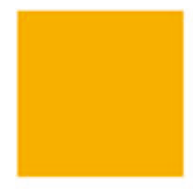

\section{Freude}

zur Übersichtsseite

\begin{tabular}{c|cccc}
$\begin{array}{c}\text { Bedeutungs- } \\
\text { erläuterung }\end{array}$ & $\begin{array}{c}\text { Kollo- } \\
\text { kationen }\end{array}$ & $\begin{array}{c}\text { Konstruk- } \\
\text { tionen }\end{array}$ & $\begin{array}{c}\text { Sinnverwandte } \\
\text { Wörter }\end{array}$ & $\begin{array}{c}\text { Gebrauchs- } \\
\text { besonderheiten }\end{array}$
\end{tabular} Grammatik

\section{i Erläuterung der Bedeutung / Funktion}

Mit Freude bezeichnet man ein Gefühl der Heiterkeit, der Fröhlichkeit und des Glücks. Das Gefühl wird z. B. ausgelöst durch einen Sachverhalt (z. B. Musik) oder durch eine Handlung (z. B. Tanzen).

Belege anzeigen »

\section{Wortklasse: Individuativum}

Abb. 5: Bedeutungserläuterung im Wortartikel Freude, Lesart 'Glücksgefühl' in elexiko

an, z.B. konsequent, Lesart 'kontinuierlich': 'Mit konsequent wird Prozessen (z.B. der Arbeit) oder Sachverhalten (z.B. der Politik) die Eigenschaft zugeschrieben, durchgängig, regelmäßig oder kontinuierlich zu sein.' (elexiko). Die paradigmatischen Partner werden manchmal auch in der Negation eingesetzt, wie bei krank, Lesart 'nicht gesund': 'Mit krank bezeichnet man den Zustand eines Lebewesens oder eines seiner Teile (z.B. eines Organs, eines Körper- oder Pflanzenteils), organisch nicht gesund zu sein. Bei Menschen und bestimmten Tieren (z.B. Haustieren) bezeichnet man mit krank auch den Zustand, geistig beeinträchtigt zu sein.' (elexiko). In den semantischen Paraphrasen der Verben finden sich oft Hinweise auf deren Rektion: erzählen, Lesart 'mitteilen': 'Mit erzählen bezeichnet man eine Sprechhandlung, bei der eine Person (einer anderen Person) Informationen mitteilt oder (sachlich) über etwas berichtet.' (elexiko). Metalinguistische Bedeutungserläuterungen, die Informationen zur Pragmatik des Lemmas beinhalten, werden standardmäßig bei fachsprachlichen Lemmata eingesetzt: Bilanz, Lesart 'Kontoabschluss': 'Mit Bilanz bezeichnet man in der Buchhaltung den Kontoabschluss, der alle Einnahmen und Ausgaben eines bestimmten Zeitraums (wie eines Geschäftsjahrs) einander gegenüberstellt.' (elexiko). Ein Beispiel für die bei Synsemantika eingesetzten Funktionsparaphrasen ist die, Lesart 'Relativpronomen': 'Mit dem femininen Relativpronomen die leitet der 
Sprecher einen Relativsatz ein und weist auf jemanden oder etwas hin, der oder das im selben Satz an anderer Stelle mit einem Nomen oder einem Pronomen bezeichnet wird (maskulin: der, neutral: das).' (elexiko).

Der erste Entwurf der semantischen Paraphrase wird in einem weiteren Arbeitsschritt durch den Lexikografen noch einmal dahingehend überprüft, ob er sachlich und sprachlich richtig sowie verständlich ist und ob die Vernetzung mit den anderen korpusbasiert gewonnenen Angaben aus den übrigen Bereichen stimmig ist: Sind die gewählten Beispiele zur Verdeutlichung typisch? Sind die in der Paraphrase benutzten sinnverwandten Wörter auch im Korpus belegt? Sind Gebrauchsbesonderheiten wie die Einstellung des Sprechers oder eine fachsprachliche Verwendung des Lemmas sowie mögliche Valenzen in die Paraphrase integriert? Auch die Korrekturleser des Wortartikels stellen sich diese und weitere Fragen zur semantischen Paraphrase erneut. So entsteht in elexiko schrittweise eine Bedeutungserläuterung in Form eines ganzen Satzes, die in ihrer Formulierung auf die satzfunktionale Klasse abgestimmt ist und mit den anderen Angabebereichen verknüpft ist. Begleitet wird die Bedeutungsparaphrase obligatorisch von ein bis drei Belegen und gegebenenfalls von einem Definitionsbeleg, Verweisen auf weitere sachliche Informationen und/ oder Illustrationen. ${ }^{37}$

\section{Ausblick}

Die semantische Paraphrase ist ein lexikografischer Angabebereich, für den es große Schwierigkeiten bereitet, ein ausführliches, allgemeingültiges methodisches Intrumentarium im Sinne der Wiegand'schen Handlungsanweisungen zusammenzustellen, sodass die Befolgung der einzelnen Handlungsschritte in einer Bedeutungserläuterung resultieren würde. Es stellt sich in diesem Zusammenhang auch die Frage, ob eine solche Methodik für die Erstellung von Bedeutungsparaphrasenangaben überhaupt notwendig und möglich ist. Denn zum einen handelt es sich beim Formulieren von semantischen Paraphrasen trotz aller redaktioneller Vorgaben und theoretischer Einflussfaktoren wie sich anbietende Typen von Bedeutungserläuterungen oder linguistische Theorien um einen kreativen Akt der Textproduktion. Dies stellt auch Wiegand fest, der das Formulieren als eine Form des Problemlösens beschreibt, die „keineswegs vollständig durch Regeln bestimmt [ist], sondern, [...] einen Spielraum für innvovative, kreative, originelle usw. Formulierungen“ (Wiegand 1984, 565)

37 Ein Definitionsbeleg beschreibt Bedeutung und Verwendung der Lesart explizit, wie im Stichwort Auge, Lesart 'Zentrum eines Wirbelsturms': „Im Zentrum des tropischen Wirbelsturms befindet sich das sogenannte Auge, in dem nahezu Windstille herrscht und das einen Durchmesser von fünfzehn bis dreißig Kilometern, in Ausnahmefällen auch von siebzig Kilometern, hat. (die tageszeitung, 31.07.1999, S. 3, Angst vor Cindy \& Bret.)“ (elexiko). 
lässt und nicht vollständig nach tradierten Formulierungsmustern ablaufen sollte. Zum anderen wäre eine konkrete Methodik wahrscheinlich nur auf ein bestimmtes Wörterbuchprojekt bezogen möglich, da jedes Wörterbuch über eigene Richtlinien zur Gestaltung der semantischen Paraphrasen verfügt. Dies bezieht sich auf die Form und den Umfang der Paraphrasen (Wortgruppen oder ganze Sätze, Grad der Ausführlichkeit und Komplexität), aber auch auf die Struktur (eingesetzte Typen der Bedeutungserläuterung).

Insofern sollte das methodische Instrumentarium für das Erstellen von Bedeutungsparaphrasenangaben eher als eine Art Werkzeugkasten der Möglichkeiten angesehen werden, aus dem sich der Lexikograf wörterbuch- und lemmaspezifisch das Geeignete heraussucht und an den speziellen Fall anpasst. Die Voraussetzung dafür ist allerdings, dass der Lexikograf die bestehenden Möglichkeiten kennt und weiß, unter welchen Bedingungen sie sich am sinnvollsten einsetzen lassen. Die einzelnen theoretischen Ansätze und Typen semantischer Paraphrasen schließen sich dabei nicht gegenseitig aus, sondern ergänzen sich im Idealfall gegenseitig (cf. Knipf-Komlósi 2000, 118).

\section{Literaturangaben}

Aitchison 1997 = Aitchison, Jean: Wörter im Kopf. Eine Einführung in das mentale Lexikon. Aus dem Englischen von Martina Wiese. Tübingen: Niemeyer, 1997 (Konzepte der Sprach- und Literaturwissenschaft 56).

Atkins/Rundell $2008=$ Atkins, B. T. Sue / Rundell, Michael (eds.): The Oxford Guide to Practical Lexicography. Oxford: Oxford University Press, 2008.

Ayto 1983 = Ayto, John R.: On specifying meaning. In: Hartmann, Reinhard R.K. (ed.): Lexicography. Principles and Practice. London: Academic Press, 1983, 89-98.

Bergenholtz/Mugdan 1990 = Bergenholtz, Henning / Mugdan, Joachim: Formen und Probleme der Datenerhebung II: Gegenwartsbezogene synchronische Wörterbücher. In: Wörterbücher. Dictionaries. Dictionnaires. Ein internationales Handbuch zur Lexikographie [...]. Hrsg. v. Hausmann, Franz Josef / Reichmann, Oskar / Wiegand, Herbert Ernst / Zgusta, Ladislav. 2. Teilbd. Berlin / New York: De Gruyter, 1990 (Handbücher zur Sprach- und Kommunikationswissenschaft 5.2), 1611-1625.

Bosque 1982 = Bosque, Ignacio: Sobre la teoría de la definición lexicográfica. In: Verba 9. 1982, $105-123$.

CCDB = CCDB - Kookkurrenzdatenbank. V3.3. Online: $\langle$ http://corpora.ids-mannheim.de/ccdb/> [2014-02-11].

COSMAS II = COSMAS II. Online: <https://cosmas2.ids-mannheim.de/cosmas2-web/> [2014-02-11]. Cramer 2011 = Cramer, Irene Magdalena: Definitionen in Wörterbuch und Text. Zur manuellen Annotation, korpusgestützten Analyse und automatischen Extraktion definitorischer Textsegmente im Kontext der computergestützten Lexikographie. Diss, TU Dortmund. Online: <http://hdl.handle.net/2003/27628> (2011) [2014-02-11].

Duden online = Duden online. $\langle$ http://www.duden.de/woerterbuch $>$ [2014-02-11]. elexiko = elexiko. In: OWID - Online Wortschatz-Informationssystem Deutsch. Hrsg. v. Institut für Deutsche Sprache, Mannheim. <www.owid.de/elexiko_/index.html> (2003ff.) [2014-02-11].

Elsen 2014 = Elsen, Hilke: Linguistische Theorien. Tübingen: Narr, 2014. 
Engelberg/Klosa/Müller-Spitzer 2009 = Engelberg, Stefan / Klosa, Annette / Müller-Spitzer, Carolin: Internet lexicography as a challenge: The Internet dictionary portal at the Institute for German Language. In: Kernerman Dictionary News (Juli), 2009, 10-19.

Geeraerts 2013 = Geeraerts, Dirk: The treatment of meaning in dictionaries and prototype theory. In: Dictionaries. An International Encyclopedia of Lexicography. Supplementary Volume: Recent Developments with Focus on Electronic and Computational Lexicography. Ed. by Gouws, Rufus / Heid, Ulrich / Schweickard, Wolfgang / Wiegand, Herbert Ernst. Berlin / Boston: De Gruyter, 2013 (Handbücher zur Sprach- und Kommunikationswissenschaft 5.4), 487-495.

grammis = grammis 2.0 - das grammatische Informationssystem des Instituts für Deutsche Sprache. Online: <http://hypermedia.ids-mannheim.de/index.html> [2014-02-11].

Hanks 1987 = Hanks, Patrick: Definitions and Explanations. In: Sinclair, John (ed.): Looking Up. An Account of the COBUILD Project in Lexical Computing. London / Glasgow: HarperCollins, 1987, 116-136.

Haß 2005a = Haß, Ulrike (Hrsg.): Grundfragen der elektronischen Lexikografie. elexiko - das Online-Informationssystem zum deutschen Wortschatz. Berlin / New York: De Gruyter, 2005 (Schriften des Instituts für Deutsche Sprache 12).

Haß 2005b = Haß, Ulrike: Das Bedeutungsspektrum. In: Haß 2005a, 163-181.

Helbig 2002 = Helbig, Gerhard: Linguistische Theorien der Moderne. Berlin: Weidler, 2002 (Germanistische Lehrbuchsammlung 19).

Herbst/Klotz 2003 = Herbst, Thomas / Klotz, Michael: Lexikografie. Paderborn: Schöningh, 2003.

Heringer 1968 = Heringer, Hans-Jürgen: ,Tag“ und ,Nacht‘. Gedanken zu einer strukturellen Lexikologie. In: Wirkendes Wort 18/4. 1968, 217-231.

Klosa 2011a $=$ Klosa, Annette: Einleitung. In: Klosa 2011b, 9-26.

Klosa 2011b = Klosa, Annette (Hrsg.): elexiko. Erfahrungsberichte aus der lexikografischen Praxis eines Internetwörterbuchs. Tübingen: Narr, 2011 (Studien zur Deutschen Sprache 55).

Klosa 2013 = Klosa, Annette: Primäre, sekundäre und tertiäre Quellen in der Lexikographie. In: Kratochvílová, Iva / Wolf, Norbert Richard (Hrsg.): Grundlagen einer sprachwissenschaftlichen Quellenkunde. Tübingen: Narr, 2013 (Studien zur Deutschen Sprache 66), 37-48.

Klosa/Storjohann 2011 = Klosa, Annette / Storjohann, Petra: Neue Überlegungen und Erfahrungen zu den lexikalischen Mitspielern. In: Klosa 2011b, 49-80.

Knipf-Komlósi 2000 = Knipf-Komlósi, Elisabeth: Prototypenbasierte Möglichkeiten in der zweisprachigen Lexikographie. In: Mangasser-Wahl, Martina (Hrsg.): Prototypentheorie in der Linguistik. Anwendungsbeispiele - Methodenreflexion - Perspektiven. Tübingen: Stauffenburg, 2000 (Stauffenburg Linguistik 10), 113-128.

Lutzeier 1985 = Lutzeier, Peter Rolf: Linguistische Semantik. Stuttgart: Metzler, 1985 (Sammlung Metzler 219).

Moerdijk $2003=$ Moerdijk, Fons: The codification of semantic information. In: Sterkenburg, Piet G. J. van (ed.): A practical guide to lexicography. Amsterdam / Philadelphia: Benjamins, 2003 (Terminology and lexicography research and practice 6), 273-296.

Müller-Spitzer 2007 = Müller-Spitzer, Carolin: Das elexiko-Portal: Ein neuer Zugang zu lexikografischen Arbeiten am Institut für Deutsche Sprache. In: Rehm, Georg / Witt, Andreas / Lemnitzer, Lothar (Hrsg.): Datenstrukturen für linguistische Ressourcen und ihre Anwendungen. Proceedings of the Biennial GLDV Conference 2007 (April 11-13, 2007, Eberhard Karls Universität Tübingen). Tübingen: Narr, 2007, 179-188.

Müller-Spitzer 2010 = Müller-Spitzer, Carolin: OWID - A dictionary net for corpus-based lexicography of contemporary German. In: Dykstra, Anne / Schoonheim, Tanneke (eds.): Proceedings of the XIV Euralex International Congress. Leeuwarden, 6-10 July 2010. Leeuwarden: Fryske Akademy, 2010, 445-452. 
Nielsen 2011 = Nielsen, Sandro: Function- and User-related Definitions in Online Dictionaries. In: Kartaškova, F. I. (ed.): Ivanovskaja leksikografičeskaja škola: tradicii i innovacii. Ivanovo School of Lexicography: Traditions and Innovations. A Festschrift in Honour of Professor Olga Karpova. Ivanovo: Ivanovo State University, 2011, 197-219.

OWID = OWID - Online-Wortschatz-Informationssystem Deutsch.

Hrsg. v. Institut für Deutsche Sprache, Mannheim. Online: 〈http://www.owid.de> (2008ff.) [2014-02-11].

Polenz 1988 = Polenz, Peter von: Deutsche Satzsemantik. Grundbegriffe des Zwischen-den-Zeilen-Lesens. 2., durchgesehene Auflage. Berlin: De Gruyter, 1988 (Sammlung Göschen 2226).

Pottier 1978 = Pottier, Bernard: Entwurf einer modernen Semantik. In: Geckeler, Horst (Hrsg.): Strukturelle Bedeutungslehre. Darmstadt: Wissenschaftliche Buchgesellschaft, 1978 (Wege der Forschung 426), 45-89.

Reichmann 2012 = Reichmann, Oskar. Historische Lexikographie. Ideen, Verwirklichungen, Reflexionen an Beispielen des Deutschen, Niederländischen und Englischen. Berlin: De Gruyter, 2012 (Studia Linguistica Germanica 111).

Rothe 2001 = Rothe, Ulrike: Das einsprachige Wörterbuch in seinem soziokulturellen Kontext. Gesellschaftliche und sprachwissenschaftliche Aspekte in der Lexikographie des Englischen und des Französischen. Tübingen: Niemeyer, 2001 (Lexicographica. Series Maior 108).

Rothe 2004 = Rothe, Ulrike: Das einsprachige Wörterbuch als Produkt von ,Kultur': Lexikographische Definitionen und Artikelbaupläne im Licht semantischer Theorien. In: Lexikografie, ihre Basis- und Nachbarwissenschaften. (Englische) Wörterbücher zwischen „common sense“ und angewandter Theorie. Herausgegeben von Thomas Herbst, Gunter Lorenz, Brigitta Mittmann und Martin Schnell. Tübingen: Niemeyer, 2001 (Lexicographica. Series Maior 118), 71-87.

Rundell 2006 = Rundell, Michael: More than One Way to Skin a Cat. Why Full-Sentence Definitions Have not Been Universally Adopted. In: Corino Elisa / Marello Carla / Onesti Cristina (eds.): Pro-ceedings of the 12th EURALEX International Congress (Atti del XIlth Congresso Internazionale di Lessicografia). EURALEX 2006, Turin, Italy, September 6th-9th, 2006. Vol. 1. Alessandria: Edizioni Dell'Orso, 2006, 323-337.

Rundell/Kilgarriff $2011=$ Rundell, Michael / Kilgarriff, Adam: Automating the creation of dictionaries. Where will it all end? In: A Taste for Corpora. A tribute to Professor Sylviane Granger. Ed. by Meunier, Fanny et al. Amsterdam / Philadelphia: Benjamins, 2011 (Studies in Corpus Linguistics 45), 257-281.

Schaeder 1981 = Schaeder, Burkhard: Lexikographie als Praxis und Theorie. Tübingen: Niemeyer, 1981 (Reihe Germanistische Linguistik 65).

Schlaefer 2009 = Schlaefer, Michael: Lexikologie und Lexikographie. Eine Einführung am Beispiel deutscher Wörterbücher. 2., durchgesehene Auflage. Berlin: Erich Schmidt, 2009 (Grundlagen der Germanistik 40).

Schmidt 1981 = Schmidt, Hartmut: Wörterbuchprobleme. Untersuchungen zu konzeptionellen Fragen der historischen Lexikographie. Tübingen: Niemeyer, 1981 (Reihe Germanistische Linguistik 34).

Storjohann 2005a = Storjohann, Petra: Semantische Paraphrasen und Kurzetikettierungen. In: Haß 2005a, 182-203.

Storjohann 2005b = Storjohann, Petra: Typische Verwendungen. In: Haß 2005a, 235-248.

Svensén 2009 = Svensén, Bo: A Handbook of Lexicography. The Theory and Practice of Dictionary-Making. Cambridge: Cambridge University Press, 2009.

Töpel 2011 = Töpel, Antje: Die semantische Paraphrase in elexiko. In: Klosa 2011b, 27-48.

Wiegand 1983 = Wiegand, Herbert Ernst: Überlegungen zu einer Theorie der lexikographischen Sprachbeschreibung. In: Symposium zur Lexikographie. Symposium on Lexicography. Proceedings of the Symposium on Lexicography, September, 1-2 1982, at the University of 
Copenhagen. Ed. by Hyldgaard-Jensen, Karl / Zettersten, Arne. Hildesheim: Georg Olms, 1983 (Germanistische Linguistik 5-6/82), 35-72.

Wiegand 1984 = Wiegand, Herbert Ernst: Prinzipien und Methoden historischer Lexikographie. In:

Sprachgeschichte. Ein Handbuch zur Geschichte der deutschen Sprache und ihrer Erforschung. Hrsg. v. Besch, Werner / Reichmann, Oskar / Sonderegger Stefan. Erster Halbbd. Berlin / New York: De Gruyter, 1984 (Handbücher zur Sprach- und Kommunikationswissenschaft 2.1), 557-620.

Wiegand 1989 = Wiegand, Herbert Ernst: Die lexikographische Definition im allgemeinen einsprachigen Wörterbuch. In: Wörterbücher. Dictionaries. Dictionnaires. Ein internationales Handbuch zur Lexikographie [...]. Hrsg. v. Hausmann, Franz Josef / Reichmann, Oskar / Wiegand, Herbert Ernst / Zgusta, Ladislav. 1. Teilbd. Berlin / New York: De Gruyter, 1989 (Handbücher zur Sprach- und Kommunikationswissenschaft 5.1), 538-588.

Wiegand 1998 = Wiegand, Herbert Ernst: Wörterbuchforschung. Untersuchungen zur Wörterbuchbenutzung, zur Theorie, Geschichte, Kritik und Automatisierung der Lexikographie. 1. Teilbd. Mit 159 Abbildungen im Text. Berlin / New York: De Gruyter, 1998.

Wiegand $2010=$ Wiegand, Herbert Ernst: Zur Methodologie der Systematischen Wörterbuchforschung: Ausgewählte Untersuchungs- und Darstellungsmethoden für die Wörterbuchform. In: Lexicographica 26. 2010, 249-330.

WLWF $=$ Wörterbuch zur Lexikographie und Wörterbuchforschung. Bd. 1, A-C. Hrsg. v. Wiegand, Herbert Ernst et al. Berlin / New York: De Gruyter, 2010.

Zgusta 1971 = Zgusta, Ladislav: Manual of Lexicography. In Cooperation with V. Černý, Z. Heřmanová-Novotná et al. Prag: Academia 1971 (Janua Linguarum. Series Maior 39). 
